\title{
Efficient Synthesis and in Silico Studies of the Benzimidazole Hybrid Scaffold with the Quinolinyloxadiazole Skeleton with Potential $\alpha$-Glucosidase Inhibitory, Anticoagulant, and Antiplatelet Activities for Type-II Diabetes Mellitus Management and Treating Thrombotic Disorders
}

\author{
S. Shashidhar Bharadwaj, ${ }^{\dagger}$ Boja Poojary, ${ }^{* \dagger \odot}$ Sharath Kumar M. Nandish, ${ }^{\ddagger}$ Jayanna Kengaiah, ${ }^{\ddagger}$
} Mugaranja P. Kirana, ${ }^{\S}$ Madan Kumar Shankar, ${ }^{\|}$Anupam J. Das, ${ }^{\perp}$ Ananda Kulal, ${ }^{\S}$ and Devaraja Sannaningaiah

\begin{abstract}
${ }^{\dagger}$ Department of Studies in Chemistry and "PURSE Lab, Mangalore University, Mangalagangotri 574 199, India
${ }^{\ddagger}$ Department of Studies and Research in Biochemistry and Centre for Bioscience and Innovation, Tumkur University, Tumkur 572103, India

${ }^{\S}$ Department of Biological Sciences, Poornaprajna Institute of Scientific Research, Bengaluru 560080, India

${ }^{\perp}$ Department of Biotechnology, School of Chemical and Biological Sciences, REVA University, Kattigenahalli Campus, Bangalore 560064, Karnataka, India
\end{abstract}

Supporting Information

ABSTRACT: The current study evaluates antidiabetic, anticoagulant, and antiplatelet activity of novel benzimidazole-containing quinolinyl oxadiazoles. These derivatives are synthesized and characterized using spectroscopy (FT-IR, ${ }^{1} \mathrm{H}$ NMR, and mass spectroscopy) and singlecrystal X-ray diffraction methods. The inhibitory effects of these compounds were evaluated by the $\alpha$-glucosidase inhibitory assay and shows the activity in the range of $\mathrm{IC}_{50}=0.66 \pm 0.05$ to $3.79 \pm 0.46$ $\mu \mathrm{g} / \mathrm{mL}$. In addition, molecular docking studies revealed that benzimidazole-containing quinolinyl oxadiazoles can correctly dock into the target receptor protein of the human intestinal $\alpha$-glucosidase, while their bioavailability/drug-likeness was predicted to be acceptable but requires further optimization. On the other hand, compound 8a and $\mathbf{8 d}$ showed anticoagulant activity as they enhanced the clotting

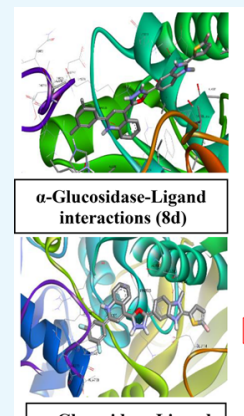

$\alpha$-Glucosidase-Ligand interactions (8a)

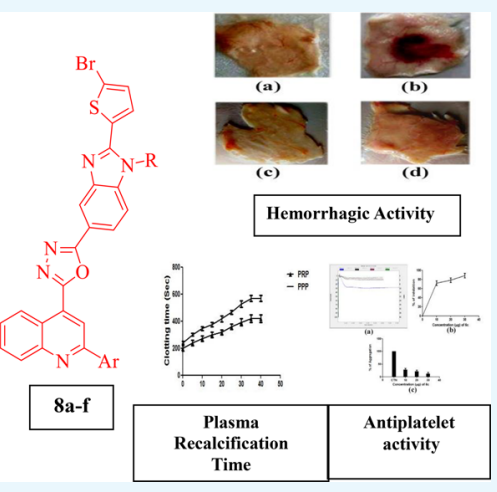
time from control $180-410$ and $180-390$ s, respectively, in platelet rich plasma and 230-460 and 230-545 s in platelet poor plasma. Furthermore, only 8a showed antiplatelet activity by inhibiting epinephrine-induced platelet aggregation, and the observed aggregation inhibition was found to be $93.4 \%$. Compounds $\mathbf{8 a - f}$ show nontoxic properties because of the non-hydrolyzing properties in the RBC cells. In addition, $\mathbf{8 a}$ and $\mathbf{8 d}$ show anti-edema and anti-hemorrhagic properties in the experimental mice. These findings reveal that benzimidazolecontaining quinolinyl oxadiazoles act as $\alpha$-glucosidase inhibitors to develop novel therapeutics for treating type-II diabetes mellitus and can act as lead molecules in drug discovery as potential antidiabetic and antithrombotic agents.

\section{INTRODUCTION}

As one of the chronic diseases, diabetes mellitus is expanding worldwide rapidly. It is characterized by high blood sugar levels for a longer period. ${ }^{1}$ This uncontrolled hyperglycemia can cause serious damage to many vital organs in the body, including the kidneys, heart, and nervous tissues. ${ }^{2,3}$ Postprandial hyperglycemia has emerged as a prominent and early defect in type- 2 diabetes and as a predictor of cardiovascular or even all-cause mortality, as well as an independent risk factor for atherosclerosis. ${ }^{4}$

The membrane-bound enzyme, $\alpha$ glucosidase, is found in the epithelium of the small intestine. It cleaves $\alpha-1,4$ glycosidic linkage at the nonreducing end of starch and disaccharides to release glucose units. Diabetes is caused with increasing glucose levels in the blood stream and relatively increases postprandial blood glucose levels. ${ }^{5-7}$ Inhibition of $\alpha$ glucosidase is involved in the reduction of glucose absorption (rate) in the intestine and further decreasing plasma glucose levels. With these properties, $\alpha$-glucosidase (therapeutic target) is used for the modulation of postprandial hyperglycemia in

Received: June 27, 2018

Accepted: September 17, 2018

Published: October 3, 2018 
type- 2 diabetes. $^{8}$ The inhibitors, acarbose, miglitol, and voglibose, used to inhibit $\alpha$-glucosidase are used to control postprandial blood glucose levels in type-2 diabetic patients. ${ }^{9,10}$

Over the past decade, $\alpha$-glucosidase inhibitors, such as acarbose, miglitol, and voglibose, have been used to decrease the postprandial blood glucose levels in type-2 diabetic patients. ${ }^{9,10}$ Furthermore, various studies show that the molecules which exhibit the $\alpha$-glucosidase inhibitor property are also used in treating cancer, HIV, virus, and tumors. ${ }^{1-13}$ Therefore, the present scenario needs a proper designing and synthesizing of better $\alpha$-glucosidase inhibitors to enrich the discipline of medicinal chemistry.

Blood plays a pivotal role in supplying micronutrients and macronutrients to different parts of the body. ${ }^{14}$ Meanwhile, blood oozing out during a vascular injury leads to many consequences. Thus, prevention of blood loss plays a major role in physiological condition and the phenomenon is termed as hemostasis, which is a highly regulated pathway. ${ }^{15}$ Some environmental and genetic factors may alter the hemostatic pathway, which leads to thrombosis. ${ }^{16}$ Thrombosis is nothing but the generation of unusual clots in arteries and veins, which is a major cause for death around the world. ${ }^{17}$ Whereas, antiplatelet and anticoagulant agents play a major role in treating thrombotic disorders. ${ }^{18}$ The present study finds the beneficial role of benzimidazole-containing quinolinyl oxadiazoles on thrombotic disorders.

Several quinoline derivatives show various pharmacological activities such as antifungal, ${ }^{19,20}$ antimalarial, ${ }^{21}$ antibacterial, ${ }^{22}$ anthelmintic, ${ }^{23,24}$ anticancer, ${ }^{25,26}$ anticonvulsant, ${ }^{27}$ anti-inflammatory, ${ }^{28}$ analgesic, ${ }^{29}$ and antihyperglycemic activities. ${ }^{30-32}$ The quinoline derivatives isolated from natural systems have been reported for potent $\alpha$-glucosidase inhibition. ${ }^{33-36}$

On the other hand, benzimidazoles have potency for the anti-inflammatory, antisplasmodic, analgesic, antihistaminic, antidiabetic, antimicrobial, antitubercular, antiproliferative, antitumor, anti-HIV-RT, anticancer, antiulcer, and cyclooxygenase inhibitor activities. ${ }^{37-46}$ The benzimidazole derivatives also exhibited $\alpha$-glucosidase ${ }^{47-49}$ as well as antidiabetic ${ }^{50}$ activity; hence, it is important to obtain novel benzimidazole derivatives as antidiabetic compounds. There are only limited studies of benzimidazole derivatives used on $\alpha$-glucosidase inhibitory (AGI) activity and cytotoxicity. ${ }^{51}$

It was reported that oxadiazoles act as a potential class of $\alpha$ glucosidase inhibitors ${ }^{34,52,53}$ and are found to have pharmacological activities such as anticancer, ${ }^{54,55}$ antimicrobial, ${ }^{56-58}$ anti-inflammatory, ${ }^{59}$ anticonvulsant, ${ }^{60}$ antioxidant, ${ }^{61}$ and antidiabetic. $^{34,62}$ The recent report on 1,3,4-oxadiazole scaffolds discusses potential antiproliferative agents. ${ }^{63-65}$

Drugs in market containing quinoline, benzimidazole, and 1,3,4-oxadiazole as a heterocycle shown in the Figure 1.

In view of the above observations in the quest to design better drugs for diabetes and thrombotic disorders in our current work, we have synthesized benzimidazole-containing quinolinyl oxadiazoles and subjected to anticoagulant, antiplatelet, and $\alpha$-glucosidase inhibition activities.

\section{RESULTS AND DISCUSSION}

Chemistry. The synthetic methods for benzimidazolecontaining quinolinyl oxadiazoles $\mathbf{8 a}-\mathbf{f}$ are illustrated in Scheme 1 . The synthesis of precursors substituted quinoline4-carboxylic acids $\mathbf{3} \mathbf{a}-\mathbf{b}$ with various acetophenones, as shown in Scheme 2. Furthermore, key intermediates $7 \mathbf{a}-\mathbf{c}$ were obtained initially from the core nucleus benzimidazole-5-

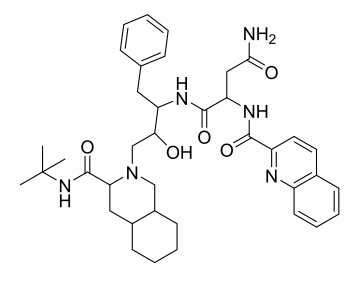

Saquinavir

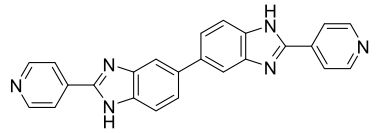

Ridinilazole

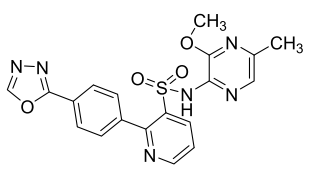

Zibotentan

Figure 1. Drugs in market containing quinoline, benzimidazole, and 1,3,4-oxadiazole moieties.

carboxylates $6 a-c$ that were efficiently synthesized by the "one pot" nitro reductive cyclization reaction between ethyl 3-nitro4-(substituted amino) benzoates $\mathbf{5 a}-\mathbf{c}$ and 5-bromothiophene2-carbaldehyde using sodium dithionite in dimethylsulfoxide. Interestingly, the "one-pot" reaction was proceeded very smoothly, in short reaction time with an excellent yield. The benzimidazole esters $\mathbf{6 a}-\mathbf{c}$ were converted into the corresponding hydrazides $7 \mathbf{a}-\mathbf{c}$ and then to highly functionalized quinolinyloxadiazoles 8a-f. All the studied compounds were synthesized by using the same procedure and characterized by spectroscopic analysis. ${ }^{66,67}$ The solid-state properties of the target compounds are listed in Supporting Information Table S1.

X-ray Diffraction Analysis and Hirshfeld Surfaces Analysis. Figure 2 describes the ORTEP of the $6 c$. The dihedral angle between rings $\mathrm{Cg} 1: \mathrm{S} 1 / \mathrm{C} 11-\mathrm{C} 14$ and $\mathrm{Cg} 4$ : $\mathrm{N} 1 / \mathrm{C} 4-\mathrm{C} 10 / \mathrm{N} 2$ is $27.4(2) 0$. Crystal structure is stabilized through $\mathrm{C} 1 \cdots \mathrm{H} 1 \mathrm{~B} \cdots \mathrm{O} 2$ intermolecular hydrogen bond in the form of 1D-infinite chain and $\mathrm{C} 16 \cdots \mathrm{H} 16 \mathrm{~A} \cdots \mathrm{Cg} 3$ (C4-C9) intermolecular interactions (Figure 3 and Table 1). The molecular arrangement within the crystal structure is shown along $a, b$, and $c$-axis (Figure 3 ). The crystal data and refinement parameters are given in Table 2.

The intercontacts in the crystal structure are analyzed and visualized using Hirshfeld surfaces and 2D fingerprint plots (Figures 4 and 5). The intercontacts found for $6 \mathrm{c}$ are $\mathrm{Br} \cdots \mathrm{C}-$ $1.5 \%, \mathrm{Br} \cdots \mathrm{H}-11.1 \%, \mathrm{Br} \cdots \mathrm{N}-2.0 \%, \mathrm{Br} \cdots \mathrm{S}-1.7 \%, \mathrm{C} \cdots \mathrm{C}-$ $1.8 \%, \mathrm{C} \cdots \mathrm{H}-18.4 \%, \mathrm{H} \cdots \mathrm{H}-39.3 \%, \mathrm{~N} \cdots \mathrm{C}-1.0 \%, \mathrm{~N} \cdots \mathrm{H}-$ $4.9 \%, \mathrm{O} \cdots \mathrm{C}-1.7 \%, \mathrm{O} \cdots \mathrm{H}-7.9 \%, \mathrm{O} \cdots \mathrm{O}-0.3 \%, \mathrm{~S} \cdots \mathrm{C}-0.4 \%$, and $\mathrm{S} \cdots \mathrm{H}-8.2 \%$. The $2 \mathrm{D}$ finger print plots of these intercontacts are shown in Figure 5. The major contributions are from $\mathrm{H} \cdots \mathrm{H}, \mathrm{C} \cdots \mathrm{H}, \mathrm{O} \cdots \mathrm{H}$, and $\mathrm{N} \cdots \mathrm{H}$ when compared to other intercontacts.

Computational Screening (Structure-Activity Relationship). The docking study was implemented using AutoDock 4.2.6 software for benzimidazole-containing quinolinyl oxadiazole derivatives and acarbose against $\mathrm{N}$-terminal domain of the human intestinal $\alpha$-glucosidase. The parameters of binding energy and binding mode of each compound were carefully analyzed. Using these parameters, hydrogen bond interactions and intermolecular interactions $(\pi-\pi)$ with the enzymes were identified. Docking studies corroborate the significant interactions of all the in vitro active benzimidazolecontaining quinolinyloxadiazole derivatives with the active site (acarbose inhibition site) of $\alpha$-glucosidase enzyme. The 
Scheme 1. Synthetic Route for the Preparation of $8 \mathrm{a}-\mathrm{f}$. Ar $=3,5-\mathrm{F}_{2} \mathrm{C}_{6} \mathrm{H}_{3}, 2,4-\mathrm{Cl}_{2} \mathrm{C}_{6} \mathrm{H}_{3} . \mathrm{R}=\mathrm{CH}_{3}, \mathrm{C}_{3} \mathrm{H}_{7}, \mathrm{C}_{4} \mathrm{H}_{9}$
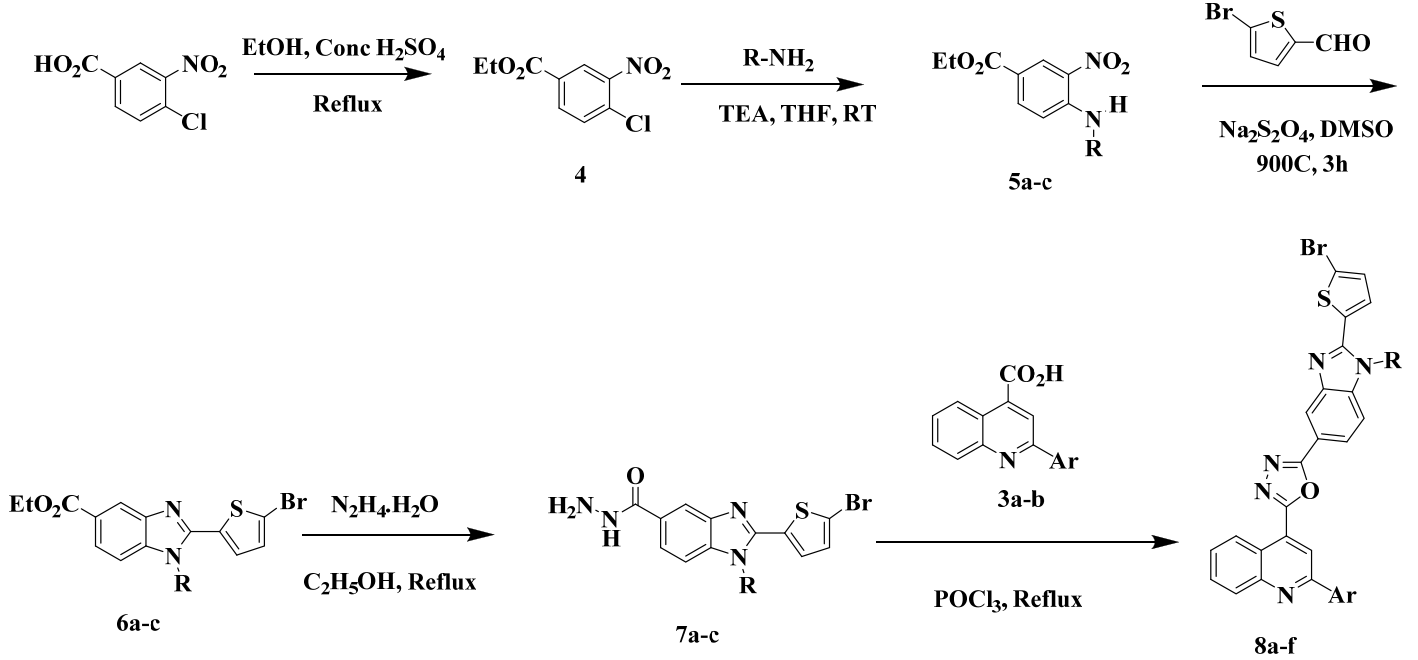

Scheme 2. Synthetic Route for the Preparation of $3 a-b$. Ar $=3,5-\mathrm{F}_{2} \mathrm{C}_{6} \mathrm{H}_{3}, 2,4-\mathrm{Cl}_{2} \mathrm{C}_{6} \mathrm{H}_{3}$<smiles>O=C1Nc2ccccc2C1=O</smiles>

1

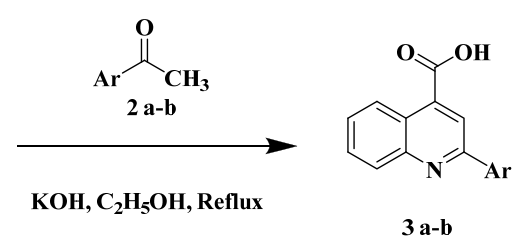

3 a-b

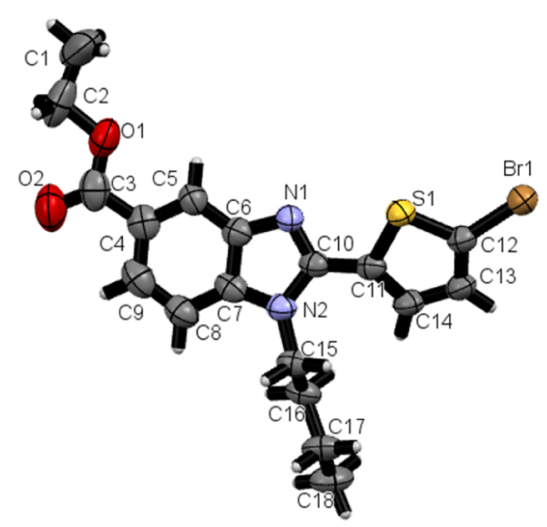

Figure 2. ORTEP diagram of the molecule $6 \mathrm{c}$ with thermal ellipsoids drawn at $50 \%$ probability.

docking scores also show positive correlation with the experimental results, in general. The docking pose of compounds 8a-f was shown in (Figure 6 and Table 3 ). The docking study predicted that the quinoline ring of compound 8d binds to enzyme forming $\pi-\pi$ stacking with the amino group of Lys776 and benzimidazole ring forms $\pi-\pi$ interaction with the amino group of Lys513 amino acid residue with the least binding energy of $-8.45 \mathrm{kcal} / \mathrm{mol}$. The second most active in this series is compound $\mathbf{8 a}$ with the least binding energy of $-8.08 \mathrm{kcal} / \mathrm{mol}$, in which quinoline ring forms $\pi-\pi$ interaction with the amino group of Lys776 while benzimidazole ring forms $\pi-\pi$ interaction with the amino

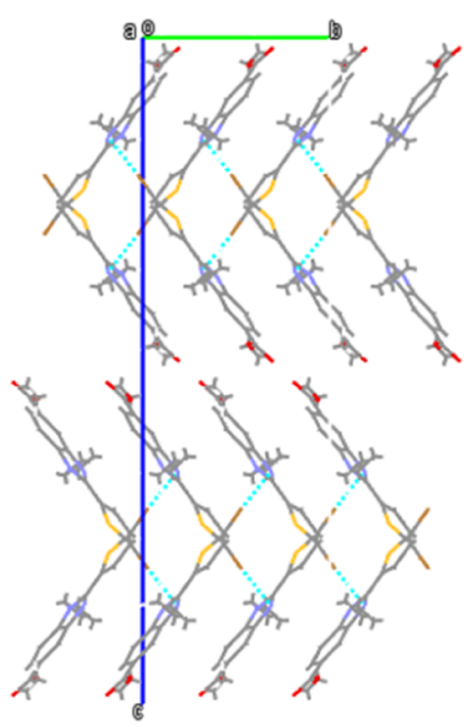

Figure 3. Molecular arrangement of the molecules viewed along the $b$-axis within the crystal structure. Dotted lines represent intermolecular hydrogen bonds.

group of Lys513. Furthermore, the benzimidazole ring of compound $\mathbf{8 b}$ forms $\pi-\pi$ stacking with the amino group of Lys 513 with the least binding energy of $-7.77 \mathrm{kcal} / \mathrm{mol}$, and in compound $8 \mathrm{f}$ with the least binding energy of $-7.5 \mathrm{kcal} / \mathrm{mol}$, the benzimidazole ring forms $\pi-\pi$ interaction with amino group of Lys513. At the binding site of a-glucosidase, the amino groups of Lys776 and Lys513 residues construct the $\pi-\pi$ stacking interaction with quinoline ring and benzimidazole ring of the ligand $\mathbf{8 c}$, respectively. However, in the ligand 8e with binding energy $-7.95 \mathrm{kcal} / \mathrm{mol}$, no interaction has been seen with amino acid residues.

In general, all the ligand molecules except $8 \mathbf{e}$ showed more promising results against $\alpha$-glucosidase compared to standard compounds. Hence, they may act as potential specific inhibitors for this target enzyme.

Biological Evaluation. Antidiabetic Activity: AGI Effect. $\alpha$-Glucosidase inhibition assay was carried out for the newly synthesized compounds $\mathbf{8 a}-\mathbf{f}$ in vitro. All the compounds 
Table 1. Intramolecular and Intermolecular Hydrogen Bonds of Compound 6c

\begin{tabular}{ccccc}
$\mathrm{D} \cdots \mathrm{H} / \mathrm{X} \cdots \mathrm{A} / \mathrm{Cg}$ & $\mathrm{D}-\mathrm{H}$ & $\mathrm{H} / \mathrm{X} \cdots \mathrm{A} / \mathrm{Cg}$ & $\mathrm{D} \cdots \mathrm{A} / \mathrm{Cg}$ & $\mathrm{D} \cdots \mathrm{H} / \mathrm{X} \cdots \mathrm{A} / \mathrm{Cg}$ \\
$\mathrm{C} 1 \cdots \mathrm{H} 1 \mathrm{~B} \cdots 2^{a}$ & 0.96 & 2.54 & $3.412(13)$ & 152 \\
$\mathrm{C} 16 \cdots \mathrm{H} 16 \mathrm{~A} \cdots \mathrm{Cg} 3^{b}$ & & 2.90 & $3.698(6)$ & 141 \\
${ }_{-}-1 / 2+x, 5 / 2-y, 1-z \cdot{ }^{b} 3 / 2-x,-1 / 2+y, z$. & & & \\
\hline
\end{tabular}

Table 2. Crystal Data and Refinement Statistics of Compound 6c

$\begin{array}{ll}\text { formula } & \mathrm{C}_{18} \mathrm{H}_{19} \mathrm{BrN}_{2} \mathrm{O}_{2} \mathrm{~S} \\ \text { formula weight } & 407.32 \\ \text { crystal system } & \text { orthorhombic } \\ \text { space group } & P b c a(\text { no. 61) } \\ a, b, c(\AA) & 11.1173(7), 9.6158(7), 34.457(2) \\ V\left(\AA^{3}\right) & 3683.5(4) \\ Z & 8 \\ D(\text { calc })\left(\mathrm{g} / \mathrm{cm}^{3}\right) & 1.469 \\ \mu(\text { Mo K } \alpha)(/ \mathrm{mm}) & 2.358 \\ F(000) & 1664 \\ \text { crystal size }(\mathrm{mm}) & 0.21 \times 0.23 \times 0.26 \\ & \text { Data Collection } \\ \text { temperature }(\mathrm{K}) & 293 \\ \text { radiation }(\AA) & \text { Mo K } \alpha 0.71073 \\ \theta \text { min-max }(\text { deg }) & 2.2,26.4 \\ \text { dataset } & -13: 13 ;-10: 12 ;-43: 43 \\ \text { tot., uniq. data, } R(\text { int }) & 37038,3761,0.187 \\ \text { observed data }[I>0.0 \sigma(I)] & 2377 \\ & \text { Refinement } \\ N_{\text {ref }} N_{\text {par }} & 3761,219 \\ R \text {, w } R_{2}, S & 0.0747,0.2225,1.05 \\ \text { max. and av. shift/error } & 0.03,0.00 \\ \text { min. and max. resd. dens. }\left(\mathrm{e} / \AA^{3}\right) & -0.63,0.83 \\ & \end{array}$
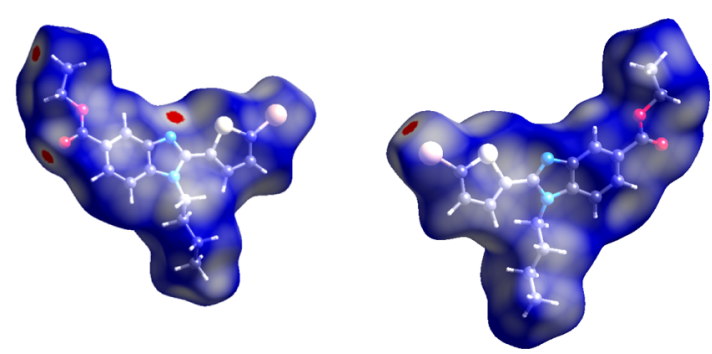

Figure 4. $d_{\text {norm }}$ mapped on Hirshfeld surface for visualizing the intercontact in different orientations. Color scale is between -0.18 au (blue) to 1.4 au (red). The ball and stick model represents the molecule orientation.

exhibited significant $\alpha$-glucosidase-inhibiting potential compared to standard acarbose $\left(\mathrm{IC}_{50}=1460.28 \pm 244.365 \mu \mathrm{g} /\right.$ $\mathrm{mL})$. It was demonstrated that compounds $8 \mathbf{a}$ and $\mathbf{8 d}$ had the best AGI activity with $\mathrm{IC}_{50}$ values $0.66 \pm 0.05$ and $0.68 \pm 0.02$ $\mu \mathrm{g} / \mathrm{mL}$ as represented in Table 4.

Plasma Recalcification Time. To pinpoint the possible role of $\mathbf{8 a}$ and $\mathbf{8 d}$ in the blood coagulation cascade, plasma recalcification time was accomplished using both human platelet rich plasma (PRP) and platelet poor plasma (PPP). Astonishingly, 8a and $\mathbf{8 d}$ displayed anticoagulant effect by enhancing the clotting time of both PRP and PPP from control $180-410,180-390$ and $230-460,230-545$ s, respectively. The supreme concentration consumed in both the cases was found to be $35 \mu \mathrm{g}$ and remain unchanged upon increased dose to $40 \mu \mathrm{g}$, Figures 7 and 8 . Coagulation factors are responsible for blood clot which is a physiological phenomenon, and the blood coagulation cascade could be activated just to arrest the bleeding in the case of injury. It involves several factors such as factor VIIa to factor XIIIa, which mainly encompasses three pathways which are intrinsic, extrinsic, and common pathways. Some genetic imbalance and some environmental factors alter the normal coagulation system, which further leads to thrombosis, a pathological phenomenon. Plentiful of anticoagulants were reported from the biological sources and nanoparticles, ${ }^{14,68-71}$ but reports available on the anticoagulants from benzimidazole-containing quinolinyl oxadiazoles are very rare.

Antiplatelet and Nontoxic Properties of $8 a-f$. Furthermore, to ascertain the role of $\mathbf{8 a}$ and $\mathbf{8 d}$ on platelets, these were analyzed for platelet aggregation assay using PRP with agonist epinephrine. Curiously, only $\mathbf{8 a}$ is able to inhibit the epinephrine-induced platelet aggregation but not the $\mathbf{8 d}$ sample. The 8a sample inhibits epinephrine-induced platelet aggregation of about $93 \%$ at the concentration of $30 \mu \mathrm{g}$ as shown in Figure 9. However, platelets are very much essential to stop bleeding along with coagulation factors. Aggregation of platelets leads to the formation of platelet plugs when blood vessels are injured. Many physiological agents act as agonists such as $\mathrm{ADP}$, epinephrine, collagen, thrombin, arachidonic acid, and thromboxane to activate platelets in order to arrest the blood by forming platelet plugs at the site injury. No control over the activation of platelets leads to pathophysiology of thrombotic disorders. Several antiplatelet agents have been identified from the biological sources, ${ }^{72,73}$ but very few are studied from synthesized compound sources.

Moreover, all the synthesized compounds of benzimidazolecontaining quinolinyl oxadiazoles $\mathbf{8 a}-\mathbf{f}$ went unhydrolyzed with RBC cells. Molecules $\mathbf{8 a}$ and $\mathbf{8 d}$ were active in preventing hemorrhage and edema in experimental mice up to the concentration level of $100 \mu \mathrm{g}$. The positive control, Daboia russelli venom, encouraged hemorrhage and edema in experimental mice, suggesting its nontoxic property as shown in Figure 10.

\section{CONCLUSIONS}

Benzimidazole-containing quinolinyloxadiazoles $8 \mathbf{a}-\mathbf{f}$ were synthesized. The novel synthesized compounds were characterized by spectral and analytical data and were screened for antidiabetic and antithrombotic activity. The results are correlated with docking studies. The molecular docking data provided positive correlation with in vitro antidiabetic activity in comparison with the standards revealed that these compounds can act as potential inhibitors. The core nucleus benzimidazole-5-carboxylates $\mathbf{6 a}-\mathbf{c}$ were efficiently synthesized by a "one pot" nitro reductive cyclization reaction between ethyl 3-nitro-4-(substituted amino) benzoates $\mathbf{5 a - c}$ and 5bromothiophene-2-carbaldehyde using sodium dithionite in dimethylsulfoxide. This "one-pot" reaction was proceeded very smoothly, in short reaction time with an excellent yield. Compounds benzimidazole-containing quinolinyloxadiazoles $\mathbf{8 a}-\mathbf{f}$ showed good binding interactions with the target enzyme 


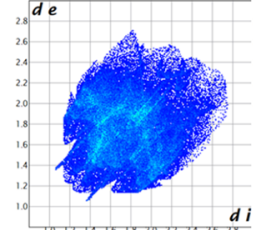

i. Full

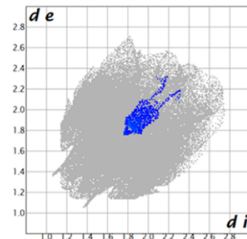

vi. C...H $-18.4 \%$

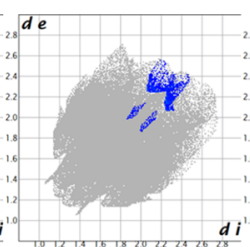

ii. $\mathrm{Br} . . \mathrm{C}-1.5 \%$

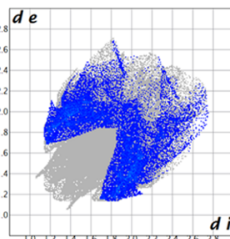

vii. H...H $-39.3 \%$

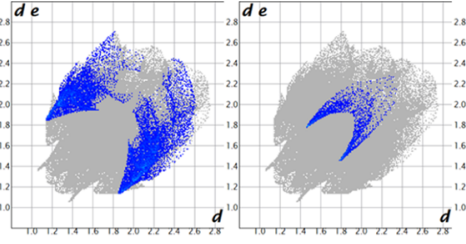

iii. Br...H- $2.0 \%$ iv. Br...S- $1.7 \%$

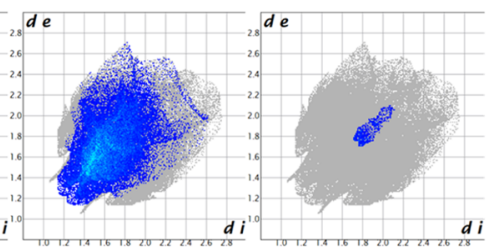

ix. $\mathbf{N}$...H $-4.9 \%$

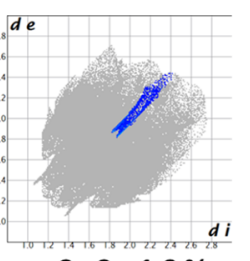

v. C...C $-1.8 \%$

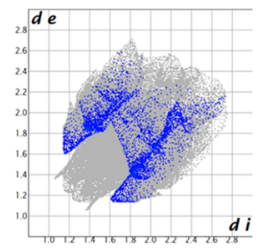

x. O...C- $1.7 \%$

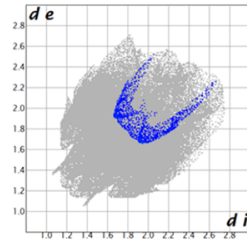

xi. 0 ...H- $7.9 \%$

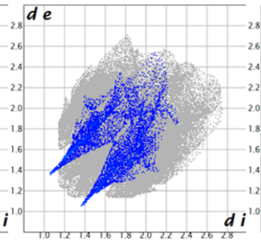

xii. $0 . . .0-0.3 \%$

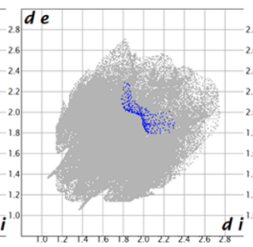

xiii. S...C $-0.4 \%$

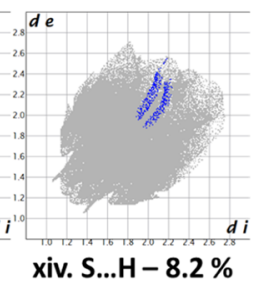

xiv. S...H $-8.2 \%$

Figure 5. Fingerprint of the title compound $\mathbf{6 c}$.

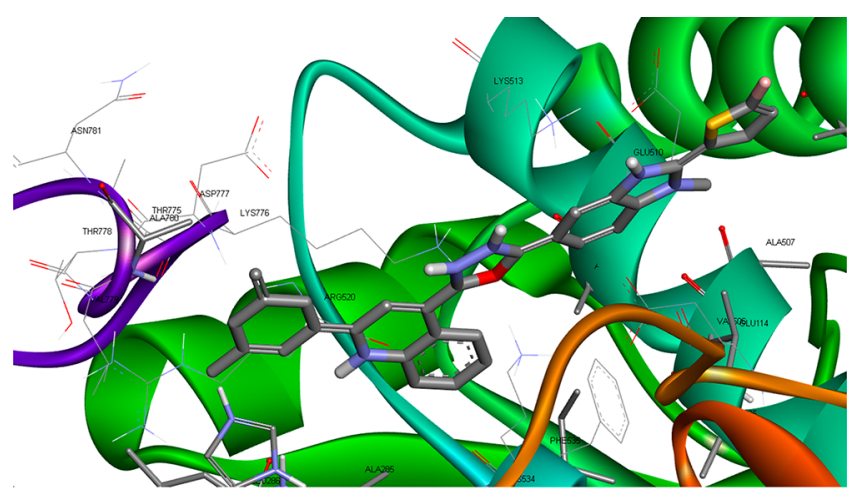

8d

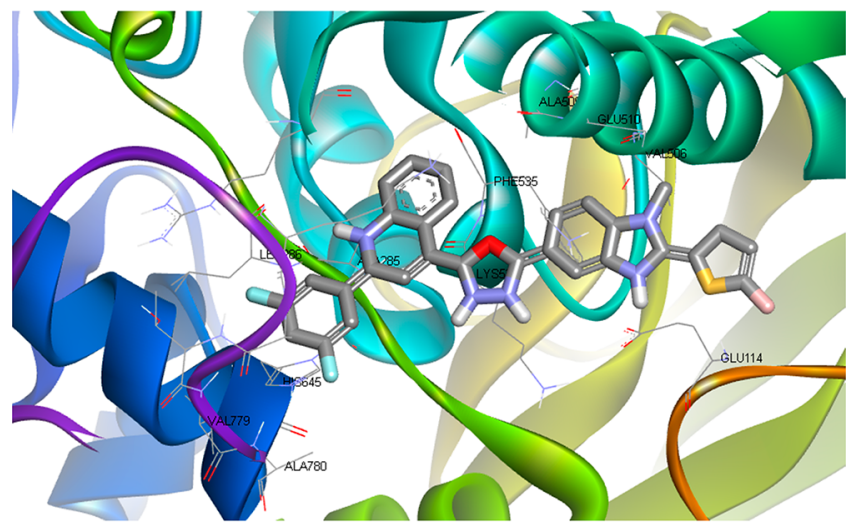

$8 \mathbf{9}$

Figure 6. Docked poses of $\alpha$-glucosidase-ligand interactions of $\mathbf{8 d}$ and 8a showing best binding affinity.

with least binding energies. Therefore, these compounds may be further evaluated and useful for diabetic treatment; on the other hand, this study for the first time demonstrates that benzimidazole-containing quinolinyl oxadiazoles $(\mathbf{8 a}-\mathbf{f})$ are responsible for anticoagulant $(\mathbf{8 a}$ and $\mathbf{8 d})$ and antiplatelet $(\mathbf{8 a})$ properties. Furthermore, they exhibit nontoxic property on RBC cells. Thus, they could play a major role in the treatment of thrombotic disorders, thus providing a new scope in drug discovery.

\section{EXPERIMENTAL SECTION}

Materials and Methods. All reagents were purchased from Sigma-Aldrich/Spectrochem, India, and used without further purification. Melting points were determined in an open capillary tube and were uncorrected. The progress of each reaction was monitored by ascending thin layer chromatography on silica gel G (Merck 1.05570.0001), visualized by UV light. The IR spectra for samples were recorded using a Shimadzu IRPrestige-21 FT-IR spectrophotometer, and the wave numbers were given in $\mathrm{cm}^{-1}$. The ${ }^{1} \mathrm{H}$ $\mathrm{NMR}$ spectra were recorded $\left(\mathrm{CDCl}_{3} / \mathrm{DMSO}-d_{6}\right.$ mixture $)$ on an Agilent $400 \mathrm{MHz}$ with an ATB probe and operated by VnmrJ software with TMS as the internal standard and Bruker; model AV 400. The X-ray intensity data were collected on a Rigaku Saturn70 diffractometer (Mo K $\alpha$ radiation) at $293 \mathrm{~K}$, and data were collected and processed using Crystal Clear. Mass spectra were recorded in a WATERS model SynaptG2 LC-mass spectrometer.

Source for Anticoagulant and Antiplatelet Activity. Fresh human blood was collected from healthy donors for the PRP and PPP.

Preparation of 2-Arylquinoline-4-carboxylic Acids (3a-b). 2-arylquinoline-4-carboxylic acid (Figure 1) was prepared according to the literature method. ${ }^{74,75}$

2-(3,5-Difluorophenyl)quinoline-4-carboxylic acid (3a): mp $230.0-232{ }^{\circ} \mathrm{C}$.

2-(3,5-Dichlorophenyl)quinoline-4-carboxylic acid (3b): mp $198-204{ }^{\circ} \mathrm{C}$.

Preparation of Ethyl 4-(Alkylamino)-3-nitrobenzoates (5a-c). Ethyl 4-chloro-3-nitro benzoate (0.01 mol) was taken in $10 \mathrm{~mL}$ of tetrahydrofuran. To this solution, primary amine $(0.015 \mathrm{~mol})$ and triethylamine $(0.03 \mathrm{~mol})$ were 
Table 3. Comparison of Predicted Binding Affinities of Active Benzimidazole-Containing Quinolinyl Oxadiazoles and Quinoline Schiff Bases and Acarbose against AGI Activity

\begin{tabular}{|c|c|c|c|c|c|c|c|}
\hline ligand & binding energy & intermol. or internal energy & internal energy & torsional energy & unbound energy & $\mathrm{H}$ bonds/AA & $\pi-\pi$ bonds/AA \\
\hline $8 d$ & -8.45 & -8.75 & $\mathbf{0}$ & 0.3 & $\mathbf{0}$ & NA & 2/Lys776, Lys513 \\
\hline $8 a$ & -8.08 & -8.38 & 0.05 & 0.3 & 0.05 & NA & 2/Lys776, Lys513 \\
\hline $8 e$ & -7.95 & -8.84 & -0.46 & 0.89 & -0.46 & NA & NA \\
\hline $8 b$ & -7.77 & -8.66 & -0.65 & 0.89 & -0.65 & NA & 1/Lys513 \\
\hline $8 f$ & -7.5 & -8.4 & -0.51 & 0.89 & -0.51 & NA & 1/Lys 513 \\
\hline $8 \mathrm{c}$ & -7.08 & -7.98 & -0.53 & 0.89 & -0.53 & NA, 3/Arg520, Lys534 & 2/Lys776, Lys513 \\
\hline acarbose & -14.38 & -18.25 & -5.52 & 3.88 & -5.52 & Val779 & NA \\
\hline
\end{tabular}

Table 4. $\mathrm{IC}_{50}$ Values of Benzimidazole-Containing Quinolinyl Oxadiazoles and Quinoline Schiff Bases for AGI Activity

$\begin{array}{lc}\text { sample } & \mathrm{IC}_{50} \text { value }(\mu \mathrm{g} / \mathrm{mL})(n=3 \pm \text { standard deviation }) \\ \mathbf{8 a} & \mathbf{0 . 6 6} \pm \mathbf{0 . 0 5} \\ \mathbf{8 b} & 2.76 \pm 0.37 \\ \mathbf{8 c} & 3.79 \pm 0.46 \\ \mathbf{8 d} & \mathbf{0 . 6 8} \pm \mathbf{0 . 0 2} \\ \mathbf{8 e} & 1.40 \pm 0.23 \\ \mathbf{8 f} & 2.81 \pm 0.24 \\ \text { acarbose } & 1460.28 \pm 244.365\end{array}$

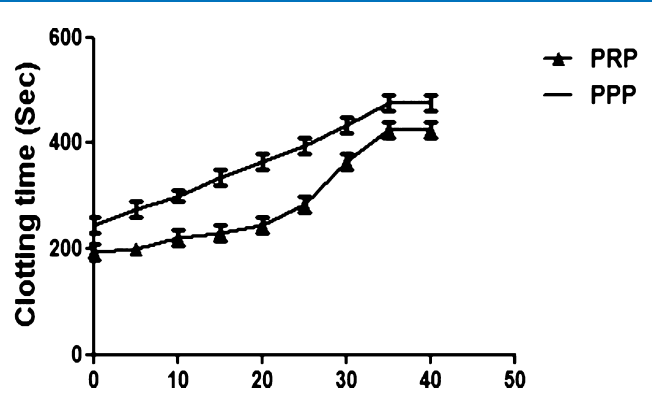

Figure 7. Plasma recalcification time. 8a $(0-40 \mu \mathrm{g})$ was preincubated with $0.2 \mathrm{~mL}$ of citrated human plasma PRP/PPP in the presence of $20 \mu \mathrm{L}$ of $10 \mathrm{mM}$ Tris- $\mathrm{HCl}$ buffer (pH 7.4) for 1 min at 37 ${ }^{\circ} \mathrm{C}$. $\mathrm{CaCl}_{2}(20 \mu \mathrm{L} ; 0.25 \mathrm{M})$ was added to the pre-incubated mixture and clotting time was recorded.

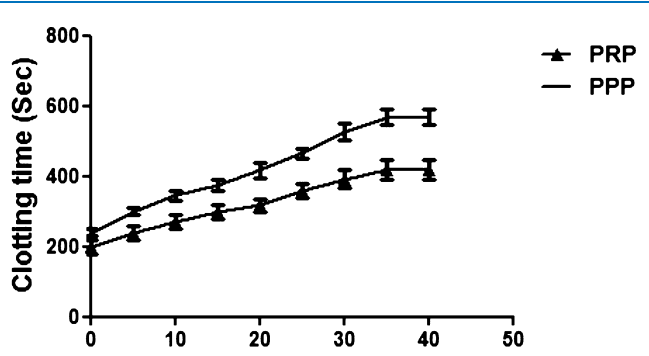

Figure 8. Plasma recalcification time. 8d $(0-40 \mu \mathrm{g})$ was preincubated with $0.2 \mathrm{~mL}$ of citrated human plasma PRP/PPP in the presence of $20 \mu \mathrm{L}$ of $10 \mathrm{mM}$ Tris- $\mathrm{HCl}$ buffer ( $\mathrm{pH} 7.4$ ) for 1 min at 37 ${ }^{\circ} \mathrm{C}$. $\mathrm{CaCl}_{2}(20 \mu \mathrm{L} ; 0.25 \mathrm{M})$ was added to the pre-incubated mixture and clotting time was recorded.

added. The reaction mixture was kept for stirring overnight at room temperature. After completion of the reaction, the reaction mixture was poured onto crushed ice. The product obtained was filtered, dried, and purified by recrystallization. Different ethyl 4-(alkylamino)-3-nitrobenzoates prepared using this method are as follows.

Ethyl 4-(Methylamino)-3-nitrobenzoate (5a): mp 98-100 ${ }^{\circ} \mathrm{C}$ (Lit. mp $\left.101{ }^{\circ} \mathrm{C}\right) .{ }^{76}$
Ethyl 4-(Propylamino)-3-nitrobenzoate (5b): $\mathrm{mp} 77-78^{\circ} \mathrm{C}$ (Lit. mp $\left.78{ }^{\circ} \mathrm{C}\right){ }^{77}$

Ethyl 4-(Butylamino)-3-nitrobenzoate (5c): mp 52-54 ${ }^{\circ} \mathrm{C}$ (Lit. mp 50-52 $\left.{ }^{\circ} \mathrm{C}\right) .^{78}$

General Procedure for the Preparation of Ethyl 2-(5Bromothiophen-2-yl)-1-alkyl-1H-benzo[d]imidazole-5carboxylates $(6 \mathbf{a}-\mathbf{c})$. Sodium dithionite (3.0 equiv) was added to a stirred solution of ethyl-4-(4-alkylamino)-3nitrobenzoate $(0.01 \mathrm{~mol})$ and 5-bromothiophene-2-carbaldehyde $(0.01 \mathrm{~mol})$ in DMSO $(20 \mathrm{~mL})$. The reaction mixture was stirred at $90{ }^{\circ} \mathrm{C}$ for $3 \mathrm{~h}$. After the completion of reaction, it was poured onto crushed ice. The solid separated was filtered off, washed with water, and dried. The crude product $6 a-c$ was recrystallized using dimethylformamide.

Ethyl 2-(5-Bromothiophen-2-yl)-1-methyl-1H-benzo[d]imidazole-5-carboxylate (6a). Yield 98\%; mp 160-165 ${ }^{\circ} \mathrm{C}$; FT IR (ATR, $\left.\nu \mathrm{cm}^{-1}\right): 3040(\mathrm{Ar}-\mathrm{H}), 2943(\mathrm{C}-\mathrm{H}), 1713(\mathrm{C}=$ O), $1616(\mathrm{C}=\mathrm{N}), 1538(\mathrm{C}=\mathrm{C}) ;{ }^{1} \mathrm{H}$ NMR (DMSO- $d_{6} \delta$ ppm): $1.43\left(\mathrm{t}, 3 \mathrm{H}, \mathrm{CH}_{3}\right), 4.04\left(\mathrm{~s}, 3 \mathrm{H}, \mathrm{CH}_{3}\right), 4.52(\mathrm{q}, 2 \mathrm{H}$, $\mathrm{CH}_{2}$ ), 7.05 (d, $1 \mathrm{H}$, Br-thienyl- $\left.\mathrm{H}, J=7.6 \mathrm{~Hz}\right), 7.28(\mathrm{~d}, 1 \mathrm{H}, \mathrm{Br}-$ thienyl- $\mathrm{H}, J=7.6 \mathrm{~Hz}), 7.48\left(\mathrm{~d}, 1 \mathrm{H}\right.$, benzimidazolyl $-\mathrm{C}_{6}-\mathrm{H}, J=$ $8.4 \mathrm{~Hz}$ ), $7.66\left(\mathrm{~d}, 1 \mathrm{H}\right.$, benzimidazolyl- $\left.\mathrm{C}_{7}-\mathrm{H}, \mathrm{J}=8.4 \mathrm{~Hz}\right), 8.03$ (s, $1 \mathrm{H}$, benzimidazolyl- $\left.\mathrm{C}_{4}-\mathrm{H}\right) ;{ }^{13} \mathrm{C} \mathrm{NMR}\left(100 \mathrm{MHz}, \mathrm{CDCl}_{3} \delta\right.$ ppm): $14.4\left(\mathrm{CH}_{3}\right), 30.1\left(\mathrm{CH}_{3}\right), 61.5\left(\mathrm{CH}_{2}\right), 110.2,110.5$, $113.0,129.5,129.8,130.9,134.2,135.1,139.4,141.5,153.9$, $167.2(\mathrm{C}=\mathrm{O})$; mass $m / z: 364.54(\mathrm{M}+\mathrm{H})^{+}, 366.79(\mathrm{M}+\mathrm{H}+$ $2)^{+}$; Anal. found (calcd) for $\mathrm{C}_{15} \mathrm{H}_{13} \mathrm{BrN}_{2} \mathrm{O}_{2} \mathrm{~S}$ (\%): C, 49.17 (49.33); H, 3.45 (3.59); 7.86 (7.67).

Ethyl 2-(5-Bromothiophen-2-yl)-1-propyl-1H-benzo[d]imidazole-5-carboxylate (6b). Yield 98\%; mp 120-124 ${ }^{\circ} \mathrm{C}$; FT IR (ATR, $\left.\nu \mathrm{cm}^{-1}\right): 3060(\mathrm{Ar}-\mathrm{H}), 2970(\mathrm{C}-\mathrm{H}), 1728(\mathrm{C}=$ O), $1629(\mathrm{C}=\mathrm{N}), 1462(\mathrm{C}=\mathrm{C}) ;{ }^{1} \mathrm{H}$ NMR (DMSO-d 6 ppm): $1.08\left(\mathrm{t}, 3 \mathrm{H}, \mathrm{CH}_{3}\right), 1.39\left(\mathrm{t}, 3 \mathrm{H}, \mathrm{CH}_{3}\right), 2.08-2.19(\mathrm{~m}$, $\left.2 \mathrm{H}, \mathrm{CH}_{2}\right), 4.35\left(\mathrm{q}, 2 \mathrm{H}, \mathrm{CH}_{2}\right), 4.42\left(\mathrm{t}, 2 \mathrm{H}, \mathrm{CH}_{2}\right), 7.13(\mathrm{~d}, 1 \mathrm{H}$, Br-thienyl-H, $J=7.6 \mathrm{~Hz}$ ), 7.35 (d, 1H, Br-thienyl-H, $J=7.6$ $\mathrm{Hz}$ ), 7.55 (d, $1 \mathrm{H}$, benzimidazolyl- $\left.\mathrm{C}_{6}-\mathrm{H}, J=8.4 \mathrm{~Hz}\right), 7.71$ (d, $1 \mathrm{H}$, benzimidazolyl- $\left.\mathrm{C}_{7}-\mathrm{H}, J=8.4 \mathrm{~Hz}\right), 8.09(\mathrm{~s}, 1 \mathrm{H}$, benzimidazolyl- $\left.\mathrm{C}_{4}-\mathrm{H}\right) ;{ }^{13} \mathrm{C}\left(100 \mathrm{MHz}, \mathrm{CDCl}_{3} \delta \mathrm{ppm}\right): 11.5$ $\left(\mathrm{CH}_{3}\right), 14.1\left(\mathrm{CH}_{3}\right), 20.9\left(\mathrm{CH}_{2}\right), 49.6\left(\mathrm{CH}_{2}\right), 61.2\left(\mathrm{CH}_{2}\right)$, $111.2,11.5,114.8,128.3,129.2,131.2,135.4,137.9,139.2$, 143.2, 154.2, $168.1(\mathrm{C}=\mathrm{O})$; mass $m / z: 393.29(\mathrm{M}+\mathrm{H})^{+}$, $395.72(\mathrm{M}+\mathrm{H}+2)^{+}$; Anal. found (calcd) for $\mathrm{C}_{17} \mathrm{H}_{17} \mathrm{BrN}_{2} \mathrm{O}_{2} \mathrm{~S}$ (\%): C, 51.75 (51.92); H, 4.17 (4.36); 7.30 (7.12).

Ethyl 2-(5-Bromothiophen-2-yl)-1-butyl-1H-benzo[d]imidazole-5-carboxylate (6c). Yield 98\%; mp 95-98 ${ }^{\circ} \mathrm{C}$; FT IR (ATR, $\left.\nu \mathrm{cm}^{-1}\right): 3090(\mathrm{Ar}-\mathrm{H}), 2985(\mathrm{C}-\mathrm{H}), 1735(\mathrm{C}=$ O), $1612(\mathrm{C}=\mathrm{N}), 1563(\mathrm{C}=\mathrm{C}) ;{ }^{1} \mathrm{H}$ NMR (DMSO- $d_{6} \delta$ ppm): $0.96\left(\mathrm{t}, 3 \mathrm{H}, \mathrm{CH}_{3}\right), 1.31\left(\mathrm{t}, 3 \mathrm{H}, \mathrm{CH}_{3}\right), 1.39-1.51(\mathrm{~m}$, $\left.2 \mathrm{H}, \mathrm{CH}_{2}\right), 1.81-1.95\left(\mathrm{~m}, 2 \mathrm{H}, \mathrm{CH}_{2}\right), 4.22\left(\mathrm{q}, 2 \mathrm{H}, \mathrm{CH}_{2}\right), 4.39$ $\left(\mathrm{t}, 2 \mathrm{H}, \mathrm{CH}_{2}\right), 7.19$ (d, $1 \mathrm{H}$, Br-thienyl-H, $J=7.6 \mathrm{~Hz}$ ), 7.45 (d, $1 \mathrm{H}$, Br-thienyl- $\mathrm{H}, J=7.6 \mathrm{~Hz}), 7.56\left(\mathrm{~d}, 1 \mathrm{H}\right.$, benzimidazolyl- $\mathrm{C}_{6}{ }^{-}$ 


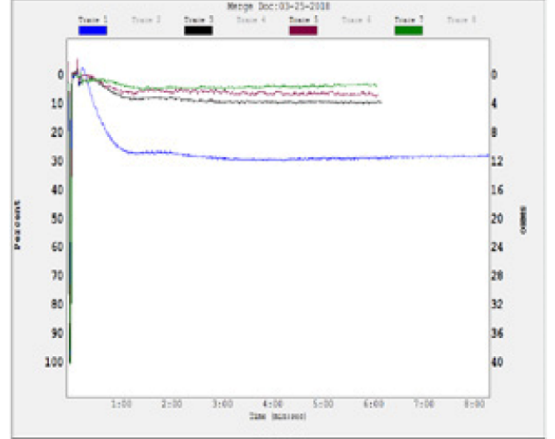

(a)

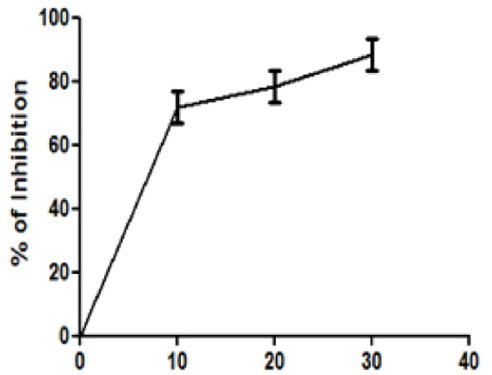

(b)

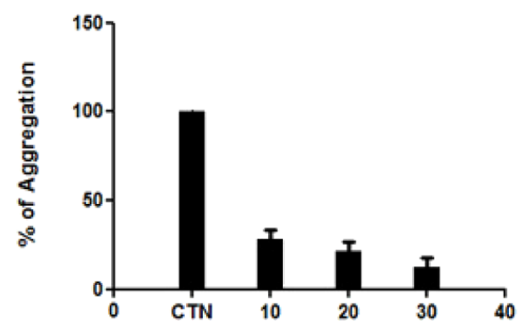

(c)

Figure 9. Platelet aggregation was initiated by adding epinephrine as an agonist of $8 \mathbf{a}$. (a) Traces of platelet aggregation: trace 1 (epinephrine 5 $\mu \mathrm{M}$ ); trace 2 (epinephrine $5 \mu \mathrm{M}+10 \mu \mathrm{g}$ of $8 \mathrm{a}$ ); trace 3 (epinephrine $5 \mu \mathrm{M}+20 \mu \mathrm{g}$ of $8 \mathrm{a}$ ); and trace 4 (epinephrine $5 \mu \mathrm{M}+30 \mu \mathrm{g}$ of $8 \mathrm{a}$ ). The values represent of three independent experiments. (b) Dose-dependent platelet aggregation inhibition \%. (c) Dose-dependent platelet aggregation $\%$.

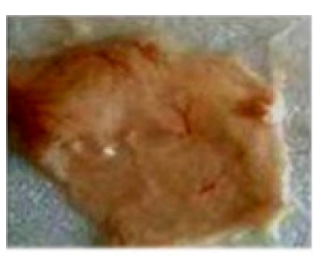

(a)

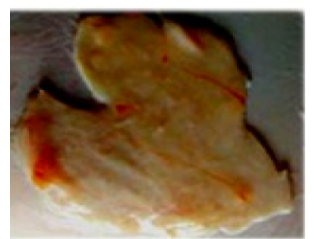

(c)

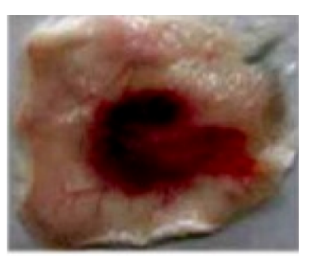

(b)

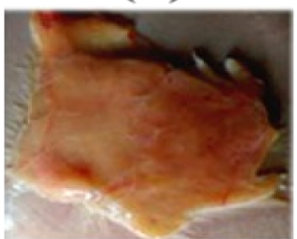

(d)
Figure 10. Dose-dependent hemorrhagic activity of $\mathbf{8 a}$ and $\mathbf{8 d}$ : (a) saline, (b) positive control $2 \mathrm{MDH}$ venom, (c) $100 \mu \mathrm{g}$ of $8 \mathrm{a}$, and (d) $100 \mu \mathrm{g}$ of $\mathbf{8 d}$ were injected independently into mice in a total volume of $50 \mu \mathrm{L}$ intradermal.

$\mathrm{H}, J=8.4 \mathrm{~Hz}$ ), 7.79 (d, $1 \mathrm{H}$, benzimidazolyl $\left.-\mathrm{C}_{7}-\mathrm{H}, J=8.4 \mathrm{~Hz}\right)$, 8.12 (s, $1 \mathrm{H}$, benzimidazolyl- $\left.\mathrm{C}_{4}-\mathrm{H}\right) ;{ }^{13} \mathrm{C}$ NMR $(100 \mathrm{MHz}$, $\left.\mathrm{CDCl}_{3} \delta \mathrm{ppm}\right): 13.9\left(\mathrm{CH}_{3}\right), 14.1\left(\mathrm{CH}_{3}\right), 21.9\left(\mathrm{CH}_{2}\right), 30.1$ $\left(\mathrm{CH}_{2}\right), 49.8\left(\mathrm{CH}_{2}\right), 62.1\left(\mathrm{CH}_{2}\right), 110.1,112.2,113.9,127.9$, 132.5, 136.4, 137.3, 138.4, 140.8, 145.2, 155.3, $168.0(\mathrm{C}=\mathrm{O})$; mass $m / z: 407.21(\mathrm{M}+\mathrm{H})^{+}, 409.53(\mathrm{M}+\mathrm{H}+2)^{+}$; Anal. found (calcd) for $\mathrm{C}_{18} \mathrm{H}_{19} \mathrm{BrN}_{2} \mathrm{O}_{2} \mathrm{~S}$ (\%): C, 53.25 (53.08); $\mathrm{H}$, 4.55 (4.70); 6.71 (6.88).

General Procedure for the Preparation of 2-(5Bromothiophen-2-yl)-1-alkyl-1H-benzo[d]imidazole-5carbohydrazide $(7 \mathrm{a}-\mathrm{c})$. A mixture of ethyl 2-(5-bromothiophen-2-yl)-1-alkyl-1H-benzo[d]imidazole-5-carboxylate (0.1 mol), hydrazine hydrate $(0.15 \mathrm{~mol})$, and $20 \mathrm{~mL}$ of ethanol was refluxed on an oil bath for $10 \mathrm{~h}$. The excess solvent was then distilled off under reduced pressure, and the concentrated solution was quenched with ice cold water. The solid separated was filtered, washed, and dried. The crude product was purified by recrystallization from ethanol.

2-(5-Bromothiophen-2-yl)-1-methyl-1H-benzo[d]imidazole-5-carbohydrazide (7a). Yield 98\%; mp >230 ${ }^{\circ} \mathrm{C}$; FT IR (ATR, $\left.\nu \mathrm{cm}^{-1}\right): 3435,3277\left(\mathrm{NH}_{2}\right), 3599(\mathrm{NH}), 3146$ $(\mathrm{Ar}-\mathrm{H}), 2947(\mathrm{C}-\mathrm{H}), 1679(\mathrm{C}=\mathrm{O}), 1663(\mathrm{C}=\mathrm{N}), 1454$ $(\mathrm{C}=\mathrm{C}), 633(\mathrm{C}-\mathrm{S}), 544(\mathrm{C}-\mathrm{Br}) ;{ }^{1} \mathrm{H}$ NMR (DMSO-d $\left.d_{6}\right) \delta$ (ppm): 4.01 (s, 3H, $\left.\mathrm{CH}_{3}\right), 4.79$ (s, 2H, $\mathrm{NH}_{2}$ ), 7.17 (d, 1H, Brthienyl- $\mathrm{H}, J=7.6 \mathrm{~Hz}), 7.40\left(\mathrm{dd}, 1 \mathrm{H}\right.$, benzimidazolyl- $\mathrm{C}_{6}-\mathrm{H}, J=$ $8.4 \mathrm{~Hz}$ ), 7.58 (dd, $1 \mathrm{H}$, benzimidazolyl- $\mathrm{C}_{7}-\mathrm{H}, J=8.4 \mathrm{~Hz}$ ), 7.67 (d, $1 \mathrm{H}$, Br-thienyl-H, $J=7.6 \mathrm{~Hz}$ ), 8.08 (s, $1 \mathrm{H}$, benzimidazolyl$\left.\mathrm{C}_{4}-\mathrm{H}\right), 9.96(\mathrm{~s}, 1 \mathrm{H}, \mathrm{NH}) ;{ }^{13} \mathrm{C} \mathrm{NMR}\left(100 \mathrm{MHz}, \mathrm{CDCl}_{3} \delta\right.$ ppm): $30.8\left(\mathrm{CH}_{3}\right), 108.8,113.0,120.4,128.7,129.2,129.8$, $134.6,134.8,135.7,141.0,152.9,165.4$; mass $m / z: 350.23(\mathrm{M}$ $+\mathrm{H})^{+}, 352.57(\mathrm{M}+\mathrm{H}+2)^{+}$; Anal. found (calcd) for $\mathrm{C}_{13} \mathrm{H}_{11} \mathrm{BrN}_{4} \mathrm{OS}$ (\%): C, 44.58 (44.46); H, 3.30 (3.16); 15.78 (15.95).

2-(5-Bromothiophen-2-yl)-1-propyl-1H-benzo[d]imidazole-5-carbohydrazide (7b). Yield 98\%; mp 140-144 ${ }^{\circ} \mathrm{C}$; FT IR (ATR, $\left.\nu \mathrm{cm}^{-1}\right): 3325,3284\left(\mathrm{NH}_{2}\right), 3511(\mathrm{NH})$, $3188(\mathrm{Ar}-\mathrm{H}), 2968(\mathrm{C}-\mathrm{H}), 1636(\mathrm{C}=\mathrm{O}), 1533(\mathrm{C}=\mathrm{N})$, $1472(\mathrm{C}=\mathrm{C}), 697(\mathrm{C}-\mathrm{S}), 561(\mathrm{C}-\mathrm{Br}) ;{ }^{1} \mathrm{H}$ NMR (DMSO-d $\delta$ ppm): $1.06\left(\mathrm{t}, 3 \mathrm{H}, \mathrm{CH}_{3}\right), 2.06-2.18\left(\mathrm{~m}, 2 \mathrm{H}, \mathrm{CH}_{2}\right), 4.31(\mathrm{t}$, $\left.2 \mathrm{H}, \mathrm{CH}_{2}\right), 4.81\left(\mathrm{~s}, 2 \mathrm{H}, \mathrm{NH}_{2}\right), 7.14(\mathrm{~d}, 1 \mathrm{H}, \mathrm{Br}$-thienyl- $\mathrm{H}, J=$ $7.6 \mathrm{~Hz}$ ), $7.44\left(\mathrm{dd}, 1 \mathrm{H}\right.$, benzimidazolyl $\left.-\mathrm{C}_{6}-\mathrm{H}, J=8.4 \mathrm{~Hz}\right), 7.55$ $(\mathrm{d}, 1 \mathrm{H}$, Br-thienyl-H, $J=7.6 \mathrm{~Hz}), 7.77(\mathrm{dd}, 1 \mathrm{H}$, benzimidazolyl- $\left.\mathrm{C}_{7}-\mathrm{H}, \mathrm{J}=8.4 \mathrm{~Hz}\right), 8.08(\mathrm{~s}, 1 \mathrm{H}$, benzimidazolyl-C $\left.\mathrm{C}_{4} \mathrm{H}\right), 10.02(\mathrm{~s}, 1 \mathrm{H}, \mathrm{NH}) ;{ }^{13} \mathrm{C} \mathrm{NMR}\left(100 \mathrm{MHz} \mathrm{CDCl}_{3}\right.$ $\delta \mathrm{ppm}): 12.5\left(\mathrm{CH}_{3}\right), 21.9\left(\mathrm{CH}_{2}\right), 49.6\left(\mathrm{CH}_{2}\right), 110.8,113.0$, $122.4,125.7,127.8,132.8,134.8,136.5,141.0,152.9,155.3$, 165.4; mass $m / z: 379.37(\mathrm{M}+\mathrm{H})^{+}, 381.71(\mathrm{M}+\mathrm{H}+2)^{+}$; 
Anal. found (calcd) for $\mathrm{C}_{15} \mathrm{H}_{15} \mathrm{BrN}_{4} \mathrm{OS}$ (\%): C, 47.67 (47.50); $\mathrm{H}, 3.81$ (3.99); 14.65 (14.77).

2-(5-Bromothiophen-2-yl)-1-butyl-1H-benzo[d]imidazole5-carbohydrazide (7c). Yield 98\%; mp >230 ${ }^{\circ} \mathrm{C}$; FT IR (ATR, $\left.\nu \mathrm{cm}^{-1}\right):$ 3345, $3298\left(\mathrm{NH}_{2}\right), 3523(\mathrm{NH}), 3159(\mathrm{Ar}-\mathrm{H}), 3009$ $(\mathrm{C}-\mathrm{H}), 1675(\mathrm{C}=\mathrm{O}), 1621(\mathrm{C}=\mathrm{N}), 1459(\mathrm{C}=\mathrm{C}), 679$ $(\mathrm{C}-\mathrm{S}), 578(\mathrm{C}-\mathrm{Br}) ;{ }^{1} \mathrm{H}$ NMR (DMSO- $\left.d_{6} \delta \mathrm{ppm}\right): 0.91(\mathrm{t}$, $\left.3 \mathrm{H}, \mathrm{CH}_{3}\right), 1.29-1.42\left(\mathrm{~m}, 2 \mathrm{H}, \mathrm{CH}_{2}\right), 1.85-1.98(\mathrm{~m}, 2 \mathrm{H}$, $\left.\mathrm{CH}_{2}\right), 4.37\left(\mathrm{t}, 2 \mathrm{H}, \mathrm{CH}_{2}\right), 4.87\left(\mathrm{~s}, 2 \mathrm{H}, \mathrm{NH}_{2}\right), 7.25(\mathrm{~d}, 1 \mathrm{H}, \mathrm{Br}-$ thienyl- $\mathrm{H}, J=7.6 \mathrm{~Hz}), 7.52\left(\mathrm{dd}, 1 \mathrm{H}\right.$, benzimidazolyl- $\mathrm{C}_{6}-\mathrm{H}, J=$ $8.4 \mathrm{~Hz}$ ), 7.68 (d, 1H, Br-thienyl-H, $J=7.6 \mathrm{~Hz}), 7.72(\mathrm{dd}, 1 \mathrm{H}$, benzimidazolyl- $\left.\mathrm{C}_{7}-\mathrm{H}, J=8.4 \mathrm{~Hz}\right), 8.16(\mathrm{~s}, 1 \mathrm{H}$, benzimidazolyl$\left.\mathrm{C}_{4}-\mathrm{H}\right), 10.14(\mathrm{~s}, 1 \mathrm{H}, \mathrm{NH}) ;{ }^{13} \mathrm{C} \mathrm{NMR}\left(100 \mathrm{MHz}, \mathrm{CDCl}_{3} \delta\right.$ ppm): $13.9\left(\mathrm{CH}_{3}\right), 20.1\left(\mathrm{CH}_{2}\right), 30.1\left(\mathrm{CH}_{2}\right), 49.6\left(\mathrm{CH}_{2}\right)$, $109.3,111.5,115.8,121.6,129.1,132.5,133.3,137.5,139.2$, 144.1, 148.2, 165.1; mass $m / z: 393.29(\mathrm{M}+\mathrm{H})^{+}, 395.68(\mathrm{M}+$ $\mathrm{H}+2)^{+}$; Anal. found (calcd) for $\mathrm{C}_{16} \mathrm{H}_{17} \mathrm{BrN}_{4} \mathrm{OS}(\%)$ : C, 48.71 (48.86); H, 4.20 (4.36); 14.44 (14.25).

General Procedure for the Preparation of 2-(2-(5Bromothiophen-2-yl)-1-alkyl-1 H-benzo[d]imidazol-5yl)-5-(2-(3,5-difluorophenyl)quinolin-4-yl)-1,3,4-oxadiazole $(8 a-f){ }^{66}$ A mixture of 2-(5-bromothiophen-2-yl)-1alkyl-1H-benzo[d]imidazole-5-carbohydrazide (1 mmol), substituted quinoline-4-carboxylic acid $(1 \mathrm{mmol})$ and phosphorus oxychloride $(2.5 \mathrm{mmol})$ was refluxed $\left(100-110{ }^{\circ} \mathrm{C}\right.$ for $6 \mathrm{~h}$ ) and the residue was quenched with ice cold water. The solid separated was processed (filtered, washed, dried, and recrystallized).

2-(2-(5-Bromothiophen-2-yl)-1-methyl-1H-benzo[d]imidazol-5-yl)-5-(2-(3,5-difluorophenyl)quinolin-4-yl)-1,3,4oxadiazole (8a). Yield 82\%; mp 214-218 ${ }^{\circ} \mathrm{C}$; FT IR (ATR, $\nu$ $\left.\mathrm{cm}^{-1}\right): 3080(\mathrm{Ar}-\mathrm{H}), 2969(\mathrm{C}-\mathrm{H}), 1604(\mathrm{C}=\mathrm{N}), 1478(\mathrm{C}=$ C), $1101(\mathrm{C}-\mathrm{F}), 692(\mathrm{C}-\mathrm{S}) ;{ }^{1} \mathrm{H}$ NMR (DMSO- $\left.d_{6} \delta \mathrm{ppm}\right)$ : $3.97\left(\mathrm{~s}, 3 \mathrm{H}, \mathrm{CH}_{3}\right), 7.38$ (overlapped $\mathrm{d}, 1 \mathrm{H}$, difluorophenyl- $\mathrm{C}_{4^{-}}$ $\mathrm{H}), 7.49$ (d, $1 \mathrm{H}$, Br-thienyl-H, $J=8.0 \mathrm{~Hz}), 7.64(\mathrm{~d}, 2 \mathrm{H}$, difluorophenyl, $J=7.2 \mathrm{~Hz}), 7.75\left(\mathrm{~d}, 1 \mathrm{H}\right.$, benzimidazolyl- $\mathrm{C}_{6}-\mathrm{H}$, $J=8.4 \mathrm{~Hz}$ ), $7.82(\mathrm{~d}, 1 \mathrm{H}$, Br-thienyl- $\mathrm{H}, J=8.0 \mathrm{~Hz}), 7.91-8.01$ $(\mathrm{m}, 2 \mathrm{H}$, quinolinyl- $\mathrm{H}), 8.17\left(\mathrm{~d}, 1 \mathrm{H}\right.$, benzimidazolyl- $\mathrm{C}_{7}-\mathrm{H}, \mathrm{J}=$ $8.4 \mathrm{~Hz}), 8.25\left(\mathrm{~s}, 1 \mathrm{H}\right.$, benzimidazolyl $\left.-\mathrm{C}_{4}-\mathrm{H}\right), 8.37(\mathrm{~d}, 1 \mathrm{H}$, quinolinyl- $\mathrm{H}, J=8.4 \mathrm{~Hz}), 8.56(\mathrm{~s}, 1 \mathrm{H}$, quinolinyl-H), $8.77(\mathrm{~d}$, $1 \mathrm{H}$, quinolinyl-H, $J=8.0 \mathrm{~Hz}) ;{ }^{13} \mathrm{C} \mathrm{NMR}\left(100 \mathrm{MHz}, \mathrm{CDCl}_{3} \delta\right.$ ppm): $30.5\left(\mathrm{CH}_{3}\right), 109.0,111.8,114.0,116.0,116.2,117.4$, $120.2,121.3,126.5,127.0,127.7,128.5,129.1,130.2,130.8$, $131.0,132.3,135.8,136.7,137.7,142.0,146.5,153.9,159.4$, $161.7,161.9,164.7,166.2$; mass $m / z: 600.41(\mathrm{M}+\mathrm{H})^{+}$, $602.29(\mathrm{M}+\mathrm{H}+2)^{+}$; Anal. found (calcd) for $\mathrm{C}_{29} \mathrm{H}_{16} \mathrm{BrF}_{2} \mathrm{~N}_{5} \mathrm{OS}$ (\%): C, 58.19 (58.01); H, 2.81 (2.69); $11.51(11.66)$.

2-(2-(5-Bromothiophen-2-yl)-1-propyl-1H-benzo[d]imidazol-5-yl)-5-(2-(3,5-difluorophenyl)quinolin-4-yl)-1,3,4oxadiazole (8b). Yield 81\%; mp 189-193 ${ }^{\circ} \mathrm{C}$; FT IR (ATR, $\nu$ $\left.\mathrm{cm}^{-1}\right)$ : $3077(\mathrm{Ar}-\mathrm{H}), 2962(\mathrm{C}-\mathrm{H}), 1606(\mathrm{C}=\mathrm{N}), 1477(\mathrm{C}=$ C), $1089(\mathrm{C}-\mathrm{F}), 606(\mathrm{C}-\mathrm{S}) ;{ }^{1} \mathrm{H}$ NMR (DMSO-d 6 . $\left.\delta \mathrm{ppm}\right)$ : $1.10\left(\mathrm{t}, 3 \mathrm{H}, \mathrm{CH}_{3}\right), 1.99-2.05\left(\mathrm{~m}, 2 \mathrm{H},-\mathrm{CH}_{2}\right), 4.48(\mathrm{t}, 2 \mathrm{H}$, $-\mathrm{CH}_{2}$ ), 6.92 (overlapped $\mathrm{d}, 1 \mathrm{H}$, difluorophenyl- $\mathrm{C}_{4}-\mathrm{H}$ ), 7.22 (d, $1 \mathrm{H}$, Br-thienyl-H, $J=7.6 \mathrm{~Hz}$ ), 7.55 (d, $1 \mathrm{H}$, Br-thienyl-H, $J$ $=7.6 \mathrm{~Hz}), 7.65-7.85(\mathrm{~m}, 2 \mathrm{H}$, quinolinyl $-\mathrm{H}), 8.15(\mathrm{~d}, 1 \mathrm{H}$, benzimidazolyl- $\left.\mathrm{C}_{7}-\mathrm{H}, \mathrm{J}=8.4 \mathrm{~Hz}\right), 8.22(\mathrm{~d}, 1 \mathrm{H}$, benzimidazolyl$\left.\mathrm{C}_{6}-\mathrm{H}, J=8.4 \mathrm{~Hz}\right), 8.33(\mathrm{~d}, 2 \mathrm{H}$, difluorophenyl- $\mathrm{H}, J=8.0 \mathrm{~Hz})$, $8.44(\mathrm{~s}, 1 \mathrm{H}$, quinolinyl- $\mathrm{H}), 8.65\left(\mathrm{~s}, 1 \mathrm{H}\right.$, benzimidazolyl- $\left.\mathrm{C}_{4}-\mathrm{H}\right)$, 8.73 (d, $1 \mathrm{H}$, quinolinyl- $\mathrm{H}, J=8.4 \mathrm{~Hz}$ ), 9.17 (d, $1 \mathrm{H}$, quinolinyl$\mathrm{H}, J=8.4 \mathrm{~Hz}) ;{ }^{13} \mathrm{C} \mathrm{NMR}\left(100 \mathrm{MHz}, \mathrm{CDCl}_{3} \delta \mathrm{ppm}\right): 12.5$ $\left(\mathrm{CH}_{3}\right), 21.9\left(\mathrm{CH}_{2}\right), 49.6\left(\mathrm{CH}_{2}\right), 108.0,110.5,113.0,117.9$,
$123.0,125.5,125.9,128.4,129.0,130.4,130.8,131.7,133.4$, $137.8,139.1,140.2,141.3,144.5,145.9,148.3,151.1,153.5$, $156.5,158.6,159.3,160.3,162.8,163.1$; mass $m / z: 628.10(\mathrm{M}$ $+\mathrm{H})^{+}, 630.25(\mathrm{M}+\mathrm{H}+2)^{+}$; Anal. found (calcd) for $\mathrm{C}_{31} \mathrm{H}_{20} \mathrm{BrF}_{2} \mathrm{~N}_{5} \mathrm{OS}$ (\%): C, 59.10 (59.24); H, 3.07 (3.21); 11.33 (11.14). HPLC purity at $254 \mathrm{~nm}, 95.2 \%$.

2-(2-(5-Bromothiophen-2-yl)-1-butyl-1H-benzo[d]imidazol-5-yl)-5-(2-(3,5-difluorophenyl)quinolin-4-yl)-1,3,4oxadiazole (8c). Yield 80\%; mp 192-194 ${ }^{\circ} \mathrm{C}$; FT IR (ATR, $\nu$ $\left.\mathrm{cm}^{-1}\right)$ : $3051(\mathrm{Ar}-\mathrm{H}), 2943(\mathrm{C}-\mathrm{H}), 1623(\mathrm{C}=\mathrm{N}), 1491(\mathrm{C}=$ C), $1123(\mathrm{C}-\mathrm{F}), 623(\mathrm{C}-\mathrm{S}) ;{ }^{1} \mathrm{H}$ NMR (DMSO- $\left.d_{6} \delta \mathrm{ppm}\right)$ : $0.96\left(\mathrm{t}, 3 \mathrm{H}, \mathrm{CH}_{3}\right), 1.27-1.35\left(\mathrm{~m}, 2 \mathrm{H},-\mathrm{CH}_{2}\right), 2.18-2.25(\mathrm{~m}$, $\left.2 \mathrm{H},-\mathrm{CH}_{2}\right), 4.58\left(\mathrm{t}, 2 \mathrm{H},-\mathrm{CH}_{2}\right), 7.34$ (overlapped $\mathrm{d}, 1 \mathrm{H}$, difluorophenyl- $\left.\mathrm{C}_{4}-\mathrm{H}\right), 7.41$ (d, $1 \mathrm{H}$, Br-thienyl- $\mathrm{H}, J=8.0 \mathrm{~Hz}$ ), $7.69(\mathrm{~d}, 2 \mathrm{H}$, difluorophenyl, $J=7.2 \mathrm{~Hz}), 7.77(\mathrm{~d}, 1 \mathrm{H}$, benzimidazolyl- $\mathrm{C}_{6}-\mathrm{H}, J=8.4 \mathrm{~Hz}$ ), 7.84 (d, $1 \mathrm{H}$, Br-thienyl-H, $J$ $=8.0 \mathrm{~Hz}), 7.94-8.09(\mathrm{~m}, 2 \mathrm{H}$, quinolinyl- $\mathrm{H}), 8.20(\mathrm{~d}, 1 \mathrm{H}$, benzimidazolyl- $\left.\mathrm{C}_{7}-\mathrm{H}, J=8.4 \mathrm{~Hz}\right), 8.29(\mathrm{~s}, 1 \mathrm{H}$, benzimidazolyl$\left.\mathrm{C}_{4}-\mathrm{H}\right), 8.40$ (d, $1 \mathrm{H}$, quinolinyl- $\left.\mathrm{H}, J=8.4 \mathrm{~Hz}\right), 8.51(\mathrm{~s}, 1 \mathrm{H}$, quinolinyl-H), $8.80(\mathrm{~d}, 1 \mathrm{H}$, quinolinyl- $\mathrm{H}, J=8.0 \mathrm{~Hz}) ;{ }^{13} \mathrm{C}$ NMR (100 MHz, $\left.\mathrm{CDCl}_{3} \delta \mathrm{ppm}\right): 13.9\left(\mathrm{CH}_{3}\right), 20.1\left(\mathrm{CH}_{2}\right)$, $30.1\left(\mathrm{CH}_{2}\right), 49.6\left(\mathrm{CH}_{2}\right), 109.0,111.1,118.5,124.6,126.1$, $126.5,129.0,130.1,131.0,131.4,132.3,134.0,138.4,140.7$, $141.8,142.9,145.1,146.5,149.9,152.7,154.1,157.1,159.2$, $160.9,161.9,163.4,165.5,165.9$; Mass $m / z: 642.21(\mathrm{M}+\mathrm{H})^{+}$, $644.39(\mathrm{M}+\mathrm{H}+2)^{+}$; Anal. found (calcd) for $\mathrm{C}_{32} \mathrm{H}_{22} \mathrm{BrF}_{2} \mathrm{~N}_{5} \mathrm{O}_{2} \mathrm{~S}$ (\%): C, 59.97 (58.82); $\mathrm{H}, 3.33$ (3.45); 10.78 (10.90).

2-(2-(5-Bromothiophen-2-yl)-1-methyl-1H-benzo[d]imidazol-5-yl)-5-(2-(2,4-dichlorophenyl)quinolin-4-yl)-1,3,4oxadiazole (8d). Yield 84\%; mp >225 ${ }^{\circ} \mathrm{C}$; FT IR (ATR, $\nu$ $\left.\mathrm{cm}^{-1}\right): 3092(\mathrm{Ar}-\mathrm{H}), 2951(\mathrm{C}-\mathrm{H}), 1655(\mathrm{C}=\mathrm{N}), 1455(\mathrm{C}=$ C), $777(\mathrm{C}-\mathrm{Cl}), 649(\mathrm{C}-\mathrm{S}) ;{ }^{1} \mathrm{H}$ NMR (DMSO- $\left.d_{6} \delta \mathrm{ppm}\right)$ : 4.08 (s, 3H, $\mathrm{CH}_{3}$ ), 7.23 (d, $1 \mathrm{H}, \mathrm{Br}$-thienyl-H, $J=8.0 \mathrm{~Hz}$ ), 7.29 (d, $1 \mathrm{H}$, Br-thienyl-H, $J=8.0 \mathrm{~Hz}$ ), 7.45 (overlapped $\mathrm{d}, 1 \mathrm{H}$, dichlorophenyl- $\left.\mathrm{C}_{3}-\mathrm{H}\right), 7.55\left(\mathrm{~s}, 1 \mathrm{H}\right.$, benzimidazolyl- $\left.\mathrm{C}_{4}-\mathrm{H}\right)$, $7.63\left(\mathrm{dd}, 2 \mathrm{H}\right.$, benzimidazolyl- $\mathrm{C}_{6}-\mathrm{H}$ and $\mathrm{C}_{7}-\mathrm{H}, \mathrm{J}=8.4 \mathrm{~Hz}$ ), 7.77-7.92 (m, 4H, dichlorophenyl- $\mathrm{H}$ and quinolinyl- $\mathrm{H}), 8.18$ $(\mathrm{s}, 1 \mathrm{H}$, quinolinyl- $\mathrm{H}), 8.54$ (d, $1 \mathrm{H}$, quinolinyl- $\mathrm{H}, J=8.4 \mathrm{~Hz}$ ), $8.75(\mathrm{~d}, 1 \mathrm{H}$, quinolinyl-H, $J=8.4 \mathrm{~Hz}) ;{ }^{13} \mathrm{C} \mathrm{NMR}(100 \mathrm{MHz}$, $\left.\mathrm{CDCl}_{3} \delta \mathrm{ppm}\right): 30.8\left(\mathrm{CH}_{3}\right), 105.0,108.2,111.6,114.4,115.5$, $120.7,121.2,122.0,122.7,123.3,123.7,124.4,124.6,125.2$, $126.0,127.7,128.4,129.5,130.5,130.9,136.2,140.7,147.1$, 148.4, 149.3, 153.1, 159.3, 160.4; mass $m / z: 631.53(\mathrm{M}+\mathrm{H})^{+}$, $633.18(\mathrm{M}+\mathrm{H}+2)^{+}$; Anal. found (calcd) for $\mathrm{C}_{29} \mathrm{H}_{16} \mathrm{BrCl}_{2} \mathrm{~N}_{5} \mathrm{OS}$ (\%): C, 55.13 (55.00); H, 2.41 (2.55); $11.22(11.06)$. HPLC purity at $254 \mathrm{~nm}, 95.5 \%$.

2-(2-(5-Bromothiophen-2-yl)-1-propyl-1H-benzo[d]imidazol-5-yl)-5-(2-(2,4-dichlorophenyl)quinolin-4-yl)-1,3,4oxadiazole (8e). Yield $83 \%$; mp $172-176{ }^{\circ} \mathrm{C}$; FT IR (ATR, $\nu$ $\left.\mathrm{cm}^{-1}\right)$ : $3061(\mathrm{Ar}-\mathrm{H}), 2981(\mathrm{C}-\mathrm{H}), 1673(\mathrm{C}=\mathrm{N}), 1463(\mathrm{C}=$ C), $759(\mathrm{C}-\mathrm{Cl}), 635(\mathrm{C}-\mathrm{S}) ;{ }^{1} \mathrm{H}$ NMR (DMSO-d $\left.d_{6} \delta \mathrm{ppm}\right)$ : $1.08\left(\mathrm{~s}, 3 \mathrm{H}, \mathrm{CH}_{3}\right), 2.29-2.38\left(\mathrm{~m}, 2 \mathrm{H},-\mathrm{CH}_{2}\right), 4.75-4.82(\mathrm{t}$, $\left.2 \mathrm{H},-\mathrm{CH}_{2}\right), 7.32(\mathrm{~d}, 1 \mathrm{H}$, Br-thienyl- $\mathrm{H}, J=8.0 \mathrm{~Hz}), 7.40$ (overlapped $\mathrm{d}, 1 \mathrm{H}$, dichlorophenyl- $\left.\mathrm{C}_{3}-\mathrm{H}\right), 7.47-7.53(\mathrm{~m}, 2 \mathrm{H}$, dichlorophenyl-H), 7.65-7.79 (m, 2H, quinolinyl- $\mathrm{H}), 7.91$ (d, $1 \mathrm{H}$, Br-thienyl-H, $J=8.0 \mathrm{~Hz}$ ), 8.19 (dd, $2 \mathrm{H}$, benzimidazolyl$\mathrm{C}_{6}-\mathrm{H}$ and $\left.\mathrm{C}_{7}-\mathrm{H}, \mathrm{J}=8.4 \mathrm{~Hz}\right), 8.28\left(\mathrm{~s}, 1 \mathrm{H}\right.$, benzimidazolyl- $\mathrm{C}_{4}-$ $\mathrm{H}), 8.37(\mathrm{~s}, 1 \mathrm{H}$, quinolinyl- $\mathrm{H}), 8.42(\mathrm{~d}, 1 \mathrm{H}$, quinolinyl- $\mathrm{H}, J=$ $8.4 \mathrm{~Hz}), 8.78(\mathrm{~d}, 1 \mathrm{H}$, quinolinyl- $\mathrm{H}, J=8.4 \mathrm{~Hz}) ;{ }^{13} \mathrm{C}$ NMR $\left(100 \mathrm{MHz}, \mathrm{CDCl}_{3} \delta \mathrm{ppm}\right): 11.5\left(\mathrm{CH}_{3}\right), 20.9\left(\mathrm{CH}_{2}\right), 48.6$ $\left(\mathrm{CH}_{2}\right), 109.8,112.0,115.4,118.2,119.3,124.5,125.0,125.7$, $126.5,126.8,127.1,127.5,128.2$, 128.6, 128.8, 129.0, 129.7, 
$132.2,133.3,134.3,134.7,140.0,142.5,144.5,151.9,157.1$, 162.2, 162.7; mass $m / z: 659.75(\mathrm{M}+\mathrm{H})^{+}, 661.29(\mathrm{M}+\mathrm{H}+$ $2)^{+}$; Anal. found (calcd) for $\mathrm{C}_{31} \mathrm{H}_{20} \mathrm{BrCl}_{2} \mathrm{~N}_{5} \mathrm{OS}$ (\%): C, 56.17 (56.30); H, 3.21 (3.05); 10.44 (10.59).

2-(2-(5-Bromothiophen-2-yl)-1-butyl-1H-benzo[d]imidazol-5-yl)-5-(2-(2,4-dichlorophenyl)quinolin-4-yl)-1,3,4oxadiazole (8f). Yield 85\%; $\mathrm{mp}>225{ }^{\circ} \mathrm{C}$; FT IR (ATR, $\nu$ $\left.\mathrm{cm}^{-1}\right)$ : 3049 (Ar-H), $2933(\mathrm{C}-\mathrm{H}), 1644(\mathrm{C}=\mathrm{N}), 1444(\mathrm{C}=$ C), $741(\mathrm{C}-\mathrm{Cl}), 658(\mathrm{C}-\mathrm{S}) ;{ }^{1} \mathrm{H}$ NMR (DMSO-d 6 . $\delta$ pm): $0.90\left(\mathrm{~s}, 3 \mathrm{H}, \mathrm{CH}_{3}\right), 1.33-1.40\left(\mathrm{~m}, 2 \mathrm{H},-\mathrm{CH}_{2}\right), 2.81-2.89(\mathrm{~m}$, $\left.2 \mathrm{H},-\mathrm{CH}_{2}\right), 4.95\left(\mathrm{t}, 2 \mathrm{H},-\mathrm{CH}_{2}\right), 7.28$ (d, $1 \mathrm{H}, \mathrm{Br}$-thienyl-H, $J$ $=8.0 \mathrm{~Hz}$ ), 7.35 (overlapped $\mathrm{d}, 1 \mathrm{H}$, dichlorophenyl- $\mathrm{C}_{3}-\mathrm{H}$ ), 7.49-7.58 (m, 2H, dichlorophenyl- $\mathrm{H}), 7.65-7.76(\mathrm{~m}, 2 \mathrm{H}$, quinolinyl- $\mathrm{H}$ ), 7.88 (dd, $2 \mathrm{H}$, benzimidazolyl- $\mathrm{C}_{6}-\mathrm{H}$ and $\mathrm{C}_{7}-\mathrm{H}, \mathrm{J}$ $=8.4 \mathrm{~Hz}), 7.97(\mathrm{~d}, 1 \mathrm{H}, \mathrm{Br}$-thienyl- $\mathrm{H}, J=8.0 \mathrm{~Hz}), 8.24(\mathrm{~s}, 1 \mathrm{H}$, benzimidazolyl- $\left.\mathrm{C}_{4}-\mathrm{H}\right), 8.33(\mathrm{~s}, 1 \mathrm{H}$, quinolinyl- $\mathrm{H}), 8.45(\mathrm{~d}, 1 \mathrm{H}$, quinolinyl- $\mathrm{H}, J=8.4 \mathrm{~Hz}), 8.81(\mathrm{~d}, 1 \mathrm{H}$, quinolinyl- $\mathrm{H}, J=8.4$ $\mathrm{Hz}) ;{ }^{13} \mathrm{C}$ NMR (100 MHz, $\left.\mathrm{CDCl}_{3} \delta \mathrm{ppm}\right): 14.5\left(\mathrm{CH}_{3}\right), 22.1$ $\left(\mathrm{CH}_{2}\right), 33.1\left(\mathrm{CH}_{2}\right), 48.6\left(\mathrm{CH}_{2}\right), 112.2,115.2,118.6,120.4$, $121.5,127.7,128.2,129.7,130.0,131.4,131.6,132.2,133.0$, $134.7,135.5,136.4,137.5,137.9,144.2,147.7,154.1,155.4$, 156.3, 160.1, 165.2, 166.3, 167.4; mass $m / z: 674.29(\mathrm{M}+\mathrm{H})^{+}$, $676.54(\mathrm{M}+\mathrm{H}+2)^{+}$; Anal. found (calcd) for $\mathrm{C}_{32} \mathrm{H}_{20} \mathrm{BrCl}_{2} \mathrm{~N}_{5} \mathrm{OS}$ (\%): C, 56.79 (56.91); H, 3.17 (3.28); 10.49 (10.37).

Single Crystal X-ray Diffraction Studies. The singlecrystal X-ray diffraction intensities collection and data process were carried out using the Rigaku Saturn 724 diffractometer (Mo K $\alpha$ radiation; $296 \mathrm{~K}$ ) and CrystalClear software, respectively. ${ }^{79}$ Direct methods were used for solving the structure (SHELXS), and refinement was made by the fullmatrix least squares method on $F^{2}$ using SHELX. ${ }^{80}$ All the nonhydrogen atoms were exhibited in the first difference Fourier map itself, in which all the hydrogen bonds were geometrically positioned $(\mathrm{C}-\mathrm{H}=0.93 \AA$, $\mathrm{O}-\mathrm{H}=0.82 \AA)$ and a riding model with $U_{\text {iso }}(\mathrm{H})=1.2 U_{\text {eq }}$ and $1.5 U_{\text {eq }}(\mathrm{O})$ was used for the refinement. The geometry of the $6 c$ is calculated using PLATON program. ${ }^{81}$ The ORTEP and packing diagrams were generated using MERCURY. ${ }^{82}$ Table 2 represents the details of crystal structure and data refinement. Figure 2 represents the ORTEP $^{82}$ of the molecule with thermal ellipsoids drawn at $50 \%$ probability.

Hirshfeld Surfaces Analysis. The Hirshfeld surfaces computational analysis was accomplished to determine the intercontacts in the crystal structure of compound $6 \mathrm{c}$ as shown in Figure 4, in which the intercontacts of the intermolecular interaction were indicated by the red colored spots over the Hirshfeld surface. The short interatomic contacts emerged by the strong hydrogen bonds were indicated by the dark red spots on the $d_{\text {norm }}$ surface, while the light red spots indicate the other intermolecular interactions. The intercontacts with respect to $d_{\mathrm{i}}$ and $d_{\mathrm{e}}$ were plotted using 2D-fingerprint plot as shown in the Figure $5 .^{74,83-86}$

Computational Screening by Molecular Docking Studies. Protein and Ligand Preparation. The N-terminal domain of $\alpha$-glucosidase protein in the human intestine (3CTT) was obtained from PDB (http://www.rcsb.org/pdb). The bound ligands, heteroatoms, and water molecules were removed from each of the protein molecules. Polar hydrogens and Gasteiger charges were assigned after merging of nonpolar hydrogen atoms. After preprocessing of the crystal structure, it was saved in the PDBQT file format using ADT. Ligands were optimized and saved in SDF format using Maestro 2D Draw (version 9.7). Using Marvin Sketch (ver 17.1.9.0; http://www. chemaxon.com), all the SDF formatted ligands were converted to the PDB format and subsequently to the PDBQT format in AutoDockTools (ADT)-1.5.6.

Molecular Docking Simulations. AutoDock 4.2.6 was used to carry out the docking simulations. A standard protocol for molecular docking was performed. To validate drug-target association, automated docking was performed with AutoDock 4.2.6. ${ }^{87}$ The grid map was set on the whole protein by AutoGrid 4 with grid dimensions to their maximum values. Ten independent genetic algorithm runs were performed for each ligand. The resulted values differing by $<2.0 \AA$ in positional root-mean-square deviation show the most favorable free energy of binding. All torsions were allowed to rotate during docking. Docked poses were visualized considering a maximum cutoff distance of $3.0 \AA$ for hydrogen bonds. Analysis and visualizations were carried out using MacPymol, ${ }^{88}$ ADT, and PoseView. ${ }^{89}$

Antidiabetic activity: AGI Effect. AGI assay was performed by the hydrolysis of chromogenic substrate $p$-nitrophenyl- $\alpha$-Dglucopyranoside (PNPG) using a previously explained method elsewhere. ${ }^{90}$ Newly synthesized compounds $8 \mathbf{8}-\mathbf{f}$ each were dissolved in DMSO to obtain $1 \mathrm{mg} / \mathrm{mL}$ stock solution. From 1 $\mathrm{mg} / \mathrm{mL}$ stock solution, further dilution was made into 0.1 , 0.005 , and $0.001 \mathrm{mg} / \mathrm{mL}$ using $0.1 \mathrm{M}$ phosphate buffer $(\mathrm{pH} 7)$ to find their $\mathrm{IC}_{50}$ values. Briefly, $100 \mu \mathrm{L}$ of reaction mixture in a 96-well microplate containing $40 \mu \mathrm{L}$ (0.0015 units) of $\alpha$ glucosidase enzyme, $50 \mu \mathrm{L}$ of compounds, and $10 \mu \mathrm{L}$ of phosphate buffer $(0.1 \mathrm{M}) \mathrm{pH} 7$ was incubated at $37{ }^{\circ} \mathrm{C}$ for 20 min. After that, $50 \mu \mathrm{L}$ of substrate ( $20 \mathrm{mM}$, PNPG) was added. The reaction mixture was further incubated at $37^{\circ} \mathrm{C}$ for $30 \mathrm{~min}$, and immediately, $100 \mu \mathrm{L}$ of sodium carbonate $(200$ $\mathrm{mM}$ ) solution was added for terminating the reaction. The reaction blank was prepared by replacing compounds and enzyme with buffer. Similarly, the control was prepared using buffer in the place of compound. Also, sample blanks were prepared for each sample replacing enzyme with buffer. The acarbose $(50 \mathrm{mg}$ ) tablets were dissolved in buffer at $10 \mathrm{mg} / \mathrm{mL}$ concentration and used as a standard. The absorbance of $p$ nitrophenol released was quantified by reading at $405 \mathrm{~nm}$ using a Thermoscientific Multiscan plate reader. The percent inhibition of $\alpha$ glucosidase enzyme was calculated using the following formula.

$$
\begin{aligned}
\operatorname{Inhibition}(\%)=[ & {\left[\text { absorbance }_{\text {control }}-\text { absorbance }_{\text {sample }_{\text {e }}}\right) } \\
& \left./ \text { absorbance }_{\text {control }}\right] \times 100
\end{aligned}
$$

The amount of sample required for the $50 \%$ inhibition of glucosidase enzyme was represented by $\mathrm{IC}_{50}$ values.

Plasma Recalcification Time. Plasma recalcification time was measured as described by Quick et al. ${ }^{91}$ Different concentrations of compounds $8 \mathrm{a}$ and $8 \mathrm{~d}(0-40 \mu \mathrm{g})$ were pre-incubated with $0.2 \mathrm{~mL}$ citrated human plasma at $37^{\circ} \mathrm{C}$ for $1 \mathrm{~min}$. Clotting was measured after the addition of $20 \mu \mathrm{L}$ $\mathrm{CaCl}_{2}(0.25 \mathrm{M})$.

Preparation of PRP and PPP. PRP and PPP were prepared using the method described by Ardlie and Han. ${ }^{92}$ The concentration of platelets in PRP was conserved as $3.1 \times 10^{8}$ platelets/mL with PPP at $37{ }^{\circ} \mathrm{C}$.

Platelet Aggregation. ${ }^{93}$ Platelet aggregation was performed in a Chronology dual channel whole blood/optical lumi aggregation system (model-700) as described by Born et al. ${ }^{93}$ PRP was pre-incubated with various concentrations of $6 \mathrm{c}(0-$ 
$15 \mu \mathrm{g}$ ) in $0.25 \mathrm{~mL}$ reaction volume mixture. The platelet aggregation process was initiated by addition of agonists independently such as ADP and epinephrine.

Direct Hemolytic Activity. Direct hemolytic activity was performed as described previously. ${ }^{18}$ Concisely, packed human erythrocytes and phosphate-buffered saline (PBS) were mixed in the ratio 1:9. Different concentrations of compounds $8 \mathbf{a}-\mathbf{f}$ $(100 \mu \mathrm{g})$ were pre-incubated with $1 \mathrm{~mL}$ of this above solution for $1 \mathrm{~h}$ at $37^{\circ} \mathrm{C}$. Then, the reaction was arrested by adding 9 $\mathrm{mL}$ of ice cold PBS and centrifuged at $1000 \mathrm{~g}$ for $10 \mathrm{~min}$ at 37 ${ }^{\circ} \mathrm{C}$. Finally, the amount of hemoglobin released was measured at $540 \mathrm{~nm}$.

Edema Inducing Activity. ${ }^{94}$ Edema inducing activity was performed as described by Sannanaik Vishwanath et al. ${ }^{94}$ Briefly, compounds $\mathbf{8 a}$ and $\mathbf{8 b}$ were injected at different doses $(10-100 \mu \mathrm{g})$ for the selected group of five mice at the right foot pads. After $1 \mathrm{~h}$, mice were anaesthetized using diethyl ether, and then, the hind-limbs were removed carefully at the ankle joint and weighed. Saline $(20 \mu \mathrm{L})$ was injected alone into the left foot serves as control. Minimum edema dose was defined as the amount of protein required to cause an edema ratio of $120 \%$.

Hemorrhagic Activity. ${ }^{95}$ Hemorrhagic activity was performed as described by Kondo et al. ${ }^{95}$ Briefly, higher concentrations of compounds $\mathbf{8 a}$ and $\mathbf{8 d}$ were injected with saline into the selected group of mice. Mice that received only saline act as the negative control whereas a group of mice that received snake venom serves as the positive control. After $3 \mathrm{~h}$, all groups of mice were anesthetized, and then, hemorrhage was noticed by removing the dorsal patch of skin surface carefully. The minimum hemorrhagic dose was defined as the amount of the compound producing $10 \mathrm{~mm}$ of hemorrhage in diameter.

Statistical Analysis. The data are presented as mean \pm SD. Statistical analyses were performed by Student's $t$-test. A significant difference between the groups was considered if $P<$ 0.01 .

\section{ASSOCIATED CONTENT}

\section{S Supporting Information}

The Supporting Information is available free of charge on the ACS Publications website at DOI: 10.1021/acsomega.8b01476.

Physical properties and structural features of target compounds; IR spectra, ${ }^{1} \mathrm{H}$ NMR spectra, RP-HPLCspectrum, and LC-MS spectrum of specific compounds (PDF)

\section{AUTHOR INFORMATION}

\section{Corresponding Author}

*E-mail: bojapoojary@gmail.com.

\section{ORCID ๑}

Boja Poojary: 0000-0002-4977-3501

Notes

The authors declare no competing financial interest.

\section{ACKNOWLEDGMENTS}

The authors are thankful to the Institute of Excellence, University of Mysore, Saif NMR Research Centre, and IISC Bangalore for providing NMR facilities and DST-PURSE Mangalore University for analytical data. The authors also thankful to Prof. Usha, Department of Biochemistry, Alva's College, Moodbidri, for her help in studying biological activity.

\section{REFERENCES}

(1) DeFronzo, R. A. Pathogenesis of Type 2 Diabetes Mellitus. Med. Clin. North Am. 2004, 88, 787-835.

(2) Deshpande, A.; Hayes, M. H.; Schootman, M. Diabetes-Related Complications. Phys. Ther. 2018, 88, 1254-1264.

(3) Cohen, A. D.; Sherf, M.; Vidavsky, L.; Vardy, D. A.; Shapiro, J.; Meyerovitch, J. Association between Psoriasis and the Metabolic Syndrome. Dermatology 2008, 216, 152-155.

(4) Boutati, E. I.; Raptis, S. A. Postprandial Hyperglycaemia in Type 2 Diabetes: Pathophysiological Aspects, Teleological Notions and Flags for Clinical Practice. Diabetes. Metab. Res. Rev. 2004, 20, S13S23.

(5) Alberti, K. G. M. M.; Taylor, R. Informed Written Consent Was Obtained from Each Subject, and the Protocol Was Approved by the Newcastle Health Authority Ethical Committee. 337. 1988, 11, 337344.

(6) Puls, W.; Keup, U.; Krause, H. P.; Thomas, G.; Hoffmeister, F. Glucosidase inhibition. Naturwissenschaften 1977, 64, 536-537.

(7) Shim, Y.-J.; Doo, H.-K.; Ahn, S.-Y.; Kim, Y.-S.; Seong, J.-K.; Park, I.-S.; Min, B.-H. Inhibitory Effect of Aqueous Extract from the Gall of Rhus Chinensis on Alpha-Glucosidase Activity and Postprandial Blood Glucose. J. Ethnopharmacol. 2003, 85, 283-287.

(8) Joshi, S. R.; Standl, E.; Tong, N.; Shah, P.; Kalra, S.; Rathod, R. Therapeutic potential of $\alpha$-glucosidase inhibitors in type 2 diabetes mellitus: an evidence-based review. Expert Opin. Pharmacother. 2015, 16, 1959-1981.

(9) Ranilla, L. G.; Kwon, Y.-I.; Apostolidis, E.; Shetty, K. Phenolic Compounds, Antioxidant Activity and in Vitro Inhibitory Potential against Key Enzymes Relevant for Hyperglycemia and Hypertension of Commonly Used Medicinal Plants, Herbs and Spices in Latin America. Bioresour. Technol. 2010, 101, 4676-4689.

(10) Toeller, M. A-Glucosidase Inhibitors in Diabetes: Efficacy in NIDDM Subjects. Eur. J. Clin. Invest. 1994, 24, 31-35.

(11) Atsumi, S.; Nosaka, C.; Ochi, Y.; Iinuma, H.; Umezawa, K. Inhibition of Experimental Metastasis by an Alpha-Glucosidase Inhibitor, 1,6-Epi-Cyclophellitol. Cancer Res. 1993, 53, 4896-4899.

(12) Gamblin, D. P.; Scanlan, E. M.; Davis, B. G. Glycoprotein Synthesis: An Update. Chem. Rev. 2009, 109, 131-163.

(13) Rawlings, A. J.; Lomas, H.; Pilling, A. W.; Lee, M. J.-R.; Alonzi, D. S.; Rountree, J. S. S.; Jenkinson, S. F.; Fleet, G. W. J.; Dwek, R. A.; Jones, J. H.; et al. Synthesis and Biological Characterisation of NovelN-Alkyl-Deoxynojirimycin $\alpha$-Glucosidase Inhibitors. ChemBioChem 2009, 10, 1101-1105.

(14) Zanetti, V. C.; da Silveira, R. B.; Dreyfuss, J. L.; Haoach, J.; Mangili, O. C.; Veiga, S. S.; Gremski, W. Morphological and Biochemical Evidence of Blood Vessel Damage and Fibrinogenolysis Triggered by Brown Spider Venom. Blood Coagul. Fibrinolysis 2002, $13,135-148$

(15) Manjappa, B.; Gangaraju, S.; Girish, K. S.; Kemparaju, K.; Gonchigar, S. J.; Shankar, R. L.; Shinde, M.; Sannaningaiah, D. Momordica Charantia Seed Extract Exhibits Strong Anticoagulant Effect by Specifically Interfering in Intrinsic Pathway of Blood Coagulation and Dissolves Fibrin Clot. Blood Coagul. Fibrinolysis 2015, 26, 191-199.

(16) Devaraja, S.; Girish, K. S.; Devaraj, V. R.; Kemparaju, K. Factor $\mathrm{Xa}$-like and fibrin(ogen)olytic activities of a serine protease from Hippasa agelenoides spider venom gland extract. J. Thromb. Thrombolysis 2010, 29, 119-126.

(17) Devaraja, S.; Girish, K. S.; Gowtham, Y. N. J.; Kemparaju, K. The Hag-protease-II is a fibrin(ogen)ase from Hippasa agelenoides spider venom gland extract: Purification, characterization and its role in hemostasis. Toxicon 2011, 57, 248-258.

(18) Nandish, S. K.; Kengaiah, J.; Ramachandraiah, C.; Shivaiah, A.; Chandramma; Girish, K. S.; Kemparaju, K.; Sannaningaiah, D. Anticoagulant, antiplatelet and fibrin clot hydrolysing activities of flax seed buffer extract. Phoog. Mag. 2018. 
(19) Musiol, R.; Jampilek, J.; Buchta, V.; Silva, L.; Niedbala, H.; Podeszwa, B.; Palka, A.; Majerz-Maniecka, K.; Oleksyn, B.; Polanski, J. Antifungal Properties of New Series of Quinoline Derivatives. Bioorg. Med. Chem. 2006, 14, 3592-3598.

(20) Musiol, R.; Serda, M.; Hensel-Bielowka, S.; Polanski, J. Quinoline-Based Antifungals. Curr. Med. Chem. 2010, 17, 1960-1973.

(21) Foley, M. Quinoline Antimalarials Mechanisms of Action and Resistance and Prospects for New Agents. Pharmacol. Ther. 1998, 79, 55-87.

(22) Sanchez, J. P.; Domagala, J. M.; Hagen, S. E.; Heifetz, C. L.; Hutt, M. P.; Nichols, J. B.; Trehan, A. K. Quinolone Antibacterial Agents. Synthesis and Structure-Activity Relationships of 8-Substituted Quinoline-3-Carboxylic Acids and 1,8-Naphthyridine-3Carboxylic Acids. J. Med. Chem. 1988, 31, 983-991.

(23) Alaimo, R. J.; Spencer, C. F.; Sheffer, J. B.; Storrin, R. J.; Hatton, C. J.; Kohls, R. E. Imidazo[4,5-f] quinolines. 4. Synthesis and anthelmintic activity of a series of imidazo[4,5-f] quinolin-9-ols. J. Med. Chem. 1978, 21, 298-300.

(24) Rossiter, S.; Péron, J.-M.; Whitfield, P. J.; Jones, K. Synthesis and Anthelmintic Properties of Arylquinolines with Activity against Drug-Resistant Nematodes. Bioorg. Med. Chem. Lett. 2005, 15, 48064808.

(25) Chen, Y.-L.; Hung, H.-M.; Lu, C.-M.; Li, K.-C.; Tzeng, C.-C. Synthesis and Anticancer Evaluation of Certain Indolo[2,3-b]Quinoline Derivatives. Bioorg. Med. Chem. 2004, 12, 6539-6546.

(26) Via, L. D.; Gia, O.; Gasparotto, V.; Ferlin, M. G. Discovery of a New Anilino-3H-Pyrrolo[3,2-f] Quinoline Derivative as Potential Anti-Cancer Agent. Eur. J. Med. Chem. 2008, 43, 429-434.

(27) Xie, Z.-F.; Chai, K.-Y.; Piao, H.-R.; Kwak, K.-C.; Quan, Z.-S. Synthesis and anticonvulsant activity of 7-alkoxyl-4,5-dihydro-[1,2,4]triazolo[4,3-a]quinolines. Bioorg. Med. Chem. Lett. 2005, 15, 48034805.

(28) Bekhit, A. A.; El-Sayed, O. A.; Aboulmagd, E.; Park, J. Y. Tetrazolo[1,5-a]Quinoline as a Potential Promising New Scaffold for the Synthesis of Novel Anti-Inflammatory and Antibacterial Agents. Eur. J. Med. Chem. 2004, 39, 249-255.

(29) Boini, K. M.; Graf, D.; Kuhl, D.; Häussinger, D.; Lang, F. SGK1 Dependence of Insulin Induced Hypokalemia. Pflugers Arch. Eur. J. Physiol. 2009, 457, 955-961.

(30) Desai, N. C.; Kotadiya, G. M.; Trivedi, A. R. Studies on Molecular Properties Prediction, Antitubercular and Antimicrobial Activities of Novel Quinoline Based Pyrimidine Motifs. Bioorg. Med. Chem. Lett. 2014, 24, 3126-3130.

(31) Sangani, C. B.; Makawana, J. A.; Zhang, X.; Teraiya, S. B.; Lin, L.; Zhu, H.-L. Design, Synthesis and Molecular Modeling of PyrazoleQuinoline-Pyridine Hybrids as a New Class of Antimicrobial and Anticancer Agents. Eur. J. Med. Chem. 2014, 76, 549-557.

(32) Taha, M.; Ismail, N. H.; Imran, S.; Wadood, A.; Rahim, F.; Ali, M.; Rehman, A. U. Novel quinoline derivatives as potent in vitro $\alpha$ glucosidase inhibitors: in silico studies and SAR predictions. Medchemcomm 2015, 6, 1826-1836.

(33) Taha, M.; Ismail, N. H.; Baharudin, M. S.; Lalani, S.; Mehboob, S.; Khan, K. M.; yousuf, S.; Siddiqui, S.; Rahim, F.; Choudhary, M. I. Synthesis crystal structure of 2-methoxybenzoylhydrazones and evaluation of their $\alpha$-glucosidase and urease inhibition potential. Med. Chem. Res. 2015, 24, 1310-1324.

(34) Taha, M.; Ismail, N. H.; Imran, S.; Rokei, M. Q. B.; Saad, S. M.; Khan, K. M. Synthesis of new oxadiazole derivatives as $\alpha$-glucosidase inhibitors. Bioorg. Med. Chem. 2015, 23, 4155-4162.

(35) Taha, M.; Naz, H.; Rasheed, S.; Ismail, N.; Rahman, A.; Yousuf, S.; Choudhary, M. Synthesis of 4-Methoxybenzoylhydrazones and Evaluation of Their Antiglycation Activity. Molecules 2014, 19, 12861301.

(36) Lee, H.-W.; Yang, J.-Y.; Lee, H.-S. Quinoline-2-carboxylic acid isolated from Ephedra pachyclada and its structural derivatives show inhibitory effects against $\alpha$-glucosidase and $\alpha$-amylase. J. Korean Soc. Appl. Biol. Chem. 2014, 57, 441-444.

(37) Merglen, A.; Theander, S.; Rubi, B.; Chaffard, G.; Wollheim, C. B.; Maechler, P. Glucose Sensitivity and Metabolism-Secretion
Coupling Studied during Two-Year Continuous Culture in INS-1E Insulinoma Cells. Endocrinology 2004, 145, 667-678.

(38) Terzioglu, N.; Gürsoy, A. Synthesis and Anticancer Evaluation of Some New Hydrazone Derivatives of 2,6-Dimethylimidazo[2,1b] $[1,3,4]$ Thiadiazole-5-Carbohydrazide. Eur. J. Med. Chem. 2003, 38, $781-786$.

(39) Gadad, A. K.; Noolvi, M. N.; Karpoormath, R. V. Synthesis and Anti-Tubercular Activity of a Series of 2-Sulfonamido/Trifluoromethyl-6-Substituted Imidazo[2,1-b]-1,3,4-Thiadiazole Derivatives. Bioorg. Med. Chem. 2004, 12, 5651-5659.

(40) Achar, K. C. S.; Hosamani, K. M.; Seetharamareddy, H. R. InVivo Analgesic and Anti-Inflammatory Activities of Newly Synthesized Benzimidazole Derivatives. Eur. J. Med. Chem. 2010, 45, 20482054.

(41) Labanauskas, L. K.; Brukštus, A. B.; Gaidelis, P. G.; Buchinskaite, V. A.; Udrenaite, É. B.; Daukšas, V. K. Synthesis and Antiinflammatory Activity of Some New 1-Acyl Derivatives of 2Methylthio-5,6-Diethoxybenzimidazole. Pharm. Chem. J. 2000, 34, $353-355$.

(42) Sondhi, S.; Bhattacharjee, G.; Jameel, R.; Shukla, R.; Raghubir, R.; Lozach, O.; Meijer, L. Antiinflammatory, analgesic and kinase inhibition activities of some acridine derivatives. Open Chem. 2004, 2, $1-15$.

(43) Garuti, L.; Roberti, M.; Pizzirani, D.; Pession, A.; Leoncini, E.; Cenci, V.; Hrelia, S. Differential Antiproliferative Activity of New Benzimidazole-4,7-Diones. Il Farmaco 2004, 59, 663-668.

(44) Kumar, D.; Jacob, M. R.; Reynolds, M. B.; Kerwin, S. M. Synthesis and Evaluation of Anticancer Benzoxazoles and Benzimidazoles Related to UK-1. Bioorg. Med. Chem. 2002, 10, 3997-4004.

(45) Paramashivappa, R.; Phani Kumar, P.; Subba Rao, P. V.; Srinivasa Rao, A. Design, Synthesis and Biological Evaluation of Benzimidazole/Benzothiazole and Benzoxazole Derivatives as Cyclooxygenase Inhibitors. Bioorg. Med. Chem. Lett. 2003, 13, 657-660.

(46) Özkay, Y.; Tunalı, Y.; Karaca, H.; Işıkdağ, I. Antimicrobial Activity and a SAR Study of Some Novel Benzimidazole Derivatives Bearing Hydrazone Moiety. Eur. J. Med. Chem. 2010, 45, 3293-3298.

(47) Taha, M.; Ismail, N. H.; Imran, S.; Mohamad, M. H.; Wadood, A.; Rahim, F.; Saad, S. M.; Rehman, A.; Khan, K. M. Synthesis, $\alpha$ glucosidase inhibitory, cytotoxicity and docking studies of 2-aryl-7methylbenzimidazoles. Bioorg. Chem. 2016, 65, 100-109.

(48) Zawawi, N. K. N. A.; Taha, M.; Ahmat, N.; Wadood, A.; Ismail, N. H.; Rahim, F.; Azam, S. S.; Abdullah, N. Benzimidazole derivatives as new $\alpha$-glucosidase inhibitors and in silico studies. Bioorg. Chem. 2016, 64, 29-36.

(49) Dinparast, L.; Valizadeh, H.; Bahadori, M. B.; Soltani, S.; Asghari, B.; Rashidi, M.-R. Design, synthesis, $\alpha$-glucosidase inhibitory activity, molecular docking and QSAR studies of benzimidazole derivatives. J. Mol. Struct. 2016, 1114, 84-94.

(50) Kwak, H. J.; Pyun, Y. M.; Kim, J. Y.; Pagire, H. S.; Kim, K. Y.; Kim, K. R.; Rhee, S. D.; Jung, W. H.; Song, J. S.; Bae, M. A.; et al. Synthesis and Biological Evaluation of Aminobenzimidazole Derivatives with a Phenylcyclohexyl Acetic Acid Group as Anti-Obesity and Anti-Diabetic Agents. Bioorg. Med. Chem. Lett. 2013, 23, 4713-4718.

(51) Kumar, J. A.; Tiwari, A. K.; Ali, A. Z.; Madhusudhana, K.; Reddy, B. S.; Ramakrishna, S.; China Raju, B. New antihyperglycemic, $\alpha$-glucosidase inhibitory, and cytotoxic derivatives of benzimidazoles. J. Enzyme Inhib. Med. Chem. 2010, 25, 80-86.

(52) Taha, M.; Ismail, N. H.; Imran, S.; Wadood, A.; Ali, M.; Rahim, F.; Khan, A. A.; Riaz, M. Novel thiosemicarbazide-oxadiazole hybrids as unprecedented inhibitors of yeast $\alpha$-glucosidase and in silico binding analysis. RSC Adv. 2016, 6, 33733-33742.

(53) Taha, M.; Ismail, N. H.; Imran, S.; Wadood, A.; Rahim, F.; Saad, S. M.; Khan, K. M.; Nasir, A. Synthesis, molecular docking and $\alpha$-glucosidase inhibition of 5-aryl-2-(6'-nitrobenzofuran-2'-yl)-1,3,4oxadiazoles. Bioorg. Chem. 2016, 66, 117-123.

(54) Bondock, S.; Adel, S.; Etman, H. A.; Badria, F. A. Synthesis and Antitumor Evaluation of Some New 1,3,4-Oxadiazole-Based Heterocycles. Eur. J. Med. Chem. 2012, 48, 192-199. 
(55) Salahuddin; Shaharyar, M.; Mazumder, A.; Ahsan, M. J. Synthesis, Characterization and Anticancer Evaluation of 2-(Naphthalen-1-Ylmethyl/Naphthalen-2-Yloxymethyl)-1-[5-(Substituted Phenyl)-[1,3,4] Oxadiazol-2-Ylmethyl]-1H-Benzimidazole. Arab. J. Chem. 2014, 7, 418-424.

(56) Aziz-ur-Rehman; Siddiqa, A.; Abbasi, M. A.; Rasool, S.; Siddiqui, S. Z.; Ahmad, I.; Afzal, S. Synthesis of Some New 5Substituted-2-((6-Chloro-3,4-Methylenedioxyphenyl)Methylthio)1,3,4-Oxadiazole Derivatives as Suitable Antibacterial Inhibitors. Bull. Fac. Pharm. (Cairo Univ.) 2015, 53, 37-43.

(57) Desai, N. C.; Dodiya, A. M.; Rajpara, K. M.; Rupala, Y. M. Synthesis and Antimicrobial Screening of 1,3,4-Oxadiazole and Clubbed Thiophene Derivatives. J. Saudi Chem. Soc. 2014, 18, 255-261.

(58) Malladi, S.; Isloor, A. M.; Peethambar, S. K.; Fun, H. K. Synthesis and Biological Evaluation of Newer Analogues of 2,5Disubstituted 1,3,4-Oxadiazole Containing Pyrazole Moiety as Antimicrobial Agents. Arab. J. Chem. 2014, 7, 1185-1191.

(59) Gadegoni, H.; Manda, S. Synthesis and Screening of Some Novel Substituted Indoles Contained 1,3,4-Oxadiazole and 1,2,4Triazole Moiety. Chin. Chem. Lett. 2013, 24, 127-130.

(60) Harish, K. P.; Mohana, K. N.; Mallesha, L.; Prasanna kumar, B. N. Synthesis of novel 1-[5-(4-methoxy-phenyl)-[1,3,4] oxadiazol-2yl]-piperazine derivatives and evaluation of their in vivo anticonvulsant activity. Eur. J. Med. Chem. 2013, 65, 276-283.

(61) Musad, E. A.; Mohamed, R.; Ali Saeed, B.; Vishwanath, B. S.; Lokanatha Rai, K. M. Synthesis and Evaluation of Antioxidant and Antibacterial Activities of New Substituted Bis(1,3,4-Oxadiazoles), 3,5-Bis(Substituted) Pyrazoles and Isoxazoles. Bioorg. Med. Chem. Lett. 2011, 21, 3536-3540.

(62) Zawawi, N. K. N. A.; Taha, M.; Ahmat, N.; Wadood, A.; Ismail, N. H.; Rahim, F.; Ali, M.; Abdullah, N.; Khan, K. M. Novel 2,5disubtituted-1,3,4-oxadiazoles with benzimidazole backbone: A new class of $\beta$-glucuronidase inhibitors and in silico studies. Bioorg. Med. Chem. 2015, 23, 3119-3125.

(63) Rashid, M.; Husain, A.; Mishra, R. Synthesis of Benzimidazoles Bearing Oxadiazole Nucleus as Anticancer Agents. Eur. J. Med. Chem. 2012, 54, 855-866.

(64) Husain, A.; Rashid, M.; Mishra, R.; Parveen, S.; Shin, D.-S.; Kumar, D. Benzimidazole Bearing Oxadiazole and TriazoloThiadiazoles Nucleus: Design and Synthesis as Anticancer Agents. Bioorg. Med. Chem. Lett. 2012, 22, 5438-5444.

(65) Puthiyapurayil, P.; Poojary, B.; Chikkanna, C.; Buridipad, S. K. Design, Synthesis and Biological Evaluation of a Novel Series of 1,3,4Oxadiazole Bearing N-Methyl-4-(Trifluoromethyl)Phenyl Pyrazole Moiety as Cytotoxic Agents. Eur. J. Med. Chem. 2012, 53, 203-210.

(66) Bharadwaj, S. S.; Poojary, B.; Madan Kumar, S.; Byrappa, K.; Nagananda, G. S.; Chaitanya, A. K.; Zaveri, K.; Yarla, N. S.; Shiralgi, Y.; Kudva, A. K.; et al. Design, Synthesis and Pharmacological Studies of Some New Quinoline Schiff Bases and 2,5-(Disubstituted-[1,3,4])Oxadiazoles. New J. Chem. 2017, 41 (). DOI: 10.1039/c6nj03913h

(67) Vasantha, K.; Basavarajaswamy, G.; Vaishali Rai, M.; Boja, P.; Pai, V. R.; Shruthi, N.; Bhat, M. Rapid 'one-pot' synthesis of a novel benzimidazole-5-carboxylate and its hydrazone derivatives as potential anti-inflammatory and antimicrobial agents. Bioorg. Med. Chem. Lett. 2015, 25, 1420-1426.

(68) Devaraja, S.; Nagaraju, S.; Mahadeswaraswamy, Y. H.; Girish, K. S.; Kemparaju, K. A Low Molecular Weight Serine Protease: Purification and Characterization from Hippasa Agelenoides (Funnel Web) Spider Venom Gland Extract. Toxicon 2008, 52, 130-138.

(69) Rachidi, S.; Aldin, E. S.; Greenberg, C.; Sachs, B.; Streiff, M.; Zeidan, A. M. The Use of Novel Oral Anticoagulants for Thromboprophylaxis after Elective Major Orthopedic Surgery. Expert Rev. Hematol. 2013, 6, 677-695.

(70) Kenawy, H. I.; Boral, I.; Bevington, A. ComplementCoagulation Cross-Talk: A Potential Mediator of the Physiological Activation of Complement by Low PH. Front. Immunol. 2015, 6, 110.
(71) Kitchen, S.; Gray, E.; Mackie, I.; Baglin, T.; Makris, M. Measurement of Non-Coumarin Anticoagulants and Their Effects on Tests of Haemostasis: Guidance from the British Committee for Standards in Haematology. Br. J. Haematol. 2014, 166, 830-841.

(72) Sim, P. J.; Wiedmers, T.; Esmonsliqb, C. T.; Weissll, H. J.; Shattil, S. J. Assembly of the Platelet Prothrombinase Complex Is Linked to Vesiculation of the Platelet Plasma Membrane. Studies in Scott syndrome: an isolated defect in platelet procoagulant activity. J. Biol. Chem. 1989, 264, 17049.

(73) Poon, M.-C.; d'Oiron, R. Recombinant activated factor VII (NovoSeven) treatment of platelet-related bleeding disorders. Blood Coagulation Fibrinolysis 2000, 11, S55-S68.

(74) Bharadwaj, S. S.; Poojary, B.; Kumar, S. M.; Byrappa, K. Synthesis, characterization, thermal analysis, crystal structure and Hirshfeld surface analysis of 2-(3, 5-difluorophenyl)quinoline-4carboxylic acid. Chem. Data Collect. 2017, 9-10, 134-142.

(75) Bharadwaj, S. S.; Kumara, K.; Poojary, B.; Yathirajan, H. S.; Byrappa, K.; Lokanath, N. K.; Kumar, S. M. Ethyl 2-(3,5Difluorophenyl)Quinoline-4-Carboxylate: A Second Triclinic Polymorph. IUCrData 2016, 1, x160739.

(76) Alkhader, M. A.; Perera, R. C.; Sinha, R. P.; Smalley, R. K. Synthesis of polynuclear heterocycles. Part 4 . Imidazo[4,5-g][3,1]benzoxazinones, imidazo[4,5-g]quinazolinones, imidazo[4,5-g]quinazolinediones, and imidazo[4,5-f]indazolinones. J. Chem. Soc., Perkin Trans. 1 1979, 1056-1062.

(77) Özden, S.; Atabey, D.; Yıldız, S.; Göker, H. Synthesis and Potent Antimicrobial Activity of Some Novel Methyl or Ethyl $1 \mathrm{H}$ Benzimidazole-5-Carboxylates Derivatives Carrying Amide or Amidine Groups. Bioorg. Med. Chem. 2005, 13, 1587-1597.

(78) Kumar, V.; Poojary, B.; Prathibha, A.; Shruthi, N. Synthesis of Some Novel 1,2-Disubstituted Benzimidazole-5-carboxylates via OnePot Method Using Sodium Dithionite and Its Effect onNDebenzylation. Synth. Commun. 2014, 44, 3414-3425.

(79) Ogi, A.; Inaba, K. X-ray thin film measurement techniques. The Rigaku J 2011, 27, 1-26.

(80) Sheldrick, G. M. A short history ofSHELX. Acta Crystallogr., Sect. A: Found. Crystallogr. 2008, 64, 112-122.

(81) Spek, A. L. PLATON, An Integrated Tool for the Analysis of the Results of a Single Crystal Structure Determination. Acta Crystallogr., Sect. A: Found. Crystallogr. 1990, 46, 34.

(82) Macrae, C. F.; Bruno, I. J.; Chisholm, J. A.; Edgington, P. R.; McCabe, P.; Pidcock, E.; Rodriguez-Monge, L.; Taylor, R.; van de Streek, J.; Wood, P. A. Mercury CSD 2.0- new features for the visualization and investigation of crystal structures. J. Appl. Crystallogr. 2008, 41, 466-470.

(83) McKinnon, J. J.; Spackman, M. A.; Mitchell, A. S. Novel Tools for Visualizing and Exploring Intermolecular Interactions in Molecular Crystals. J. Acta. Crystallogr. 2004, 60, 627-668.

(84) Spackman, M. A.; Jayatilaka, D. Hirshfeld Surface Analysis. CrystEngComm 2009, 11, 19-32.

(85) Wood, P. A.; McKinnon, J. J.; Parsons, S.; Pidcock, E.; Spackman, M. A. Analysis of the Compression of Molecular Crystal Structures Using Hirshfeld Surfaces. CrystEngComm 2008, 10, 368376.

(86) Kumar, S. M.; Manjunath, B. C.; Lingaraju, G. S.; Abdoh, M. M. M.; Sadashiva, M. P.; Lokanath, N. K. A Hirshfeld Surface Analysis and Crystal Structure of 2'-[1-(2-Fluoro-Phenyl)-1H-tetrazol-5-Yl]-4Methoxy-Biphenyl-2-Carbaldehyde. Cryst. Struct. Theory Appl. 2013, 2, 124-131.

(87) Buzko, O. V.; Bishop, A. C.; Shokat, K. M. Modified AutoDock for Accurate Docking of Protein Kinase Inhibitors. J. Comput. Aided. Mol. Des. 2002, 16, 113-127.

(88) Adams, P. D.; Afonine, P. V.; Bunkóczi, G.; Chen, V. B.; Davis, I. W.; Echols, N.; Headd, J. J.; Hung, L.-W.; Kapral, G. J.; GrosseKunstleve, R. W.; et al. PHENIX: A Comprehensive Python-Based System for Macromolecular Structure Solution. Acta Crystallogr., Sect. D: Biol. Crystallogr. 2010, 66, 213-221. 
(89) Stierand, K.; Rarey, M. From Modeling to Medicinal Chemistry: Automatic Generation of Two-Dimensional Complex Diagrams. ChemMedChem 2007, 2, 853-860.

(90) Tao, Y.; Zhang, Y.; Cheng, Y.; Wang, Y. Rapid screening and identification of $\alpha$-glucosidase inhibitors from mulberry leaves using enzyme-immobilized magnetic beads coupled with HPLC/MS and NMR. Biomed. Chromatogr. 2013, 27, 148-155.

(91) Quick, A. J.; Stanley-Brown, M.; Bancroft, F. W. A Study of the Coagulation Defect in Hemophilia and in Jaundice. Am. J. Med. Sci. 1935, 190, 501-510.

(92) Ardlie, N. G.; Han, P. Enzymatic Basis for Platelet Aggregation and Release: The Significance of the 'Platelet Atmosphere' and the Relationship between Platelet Function and Blood Coagulation. Br. J. Haematol. 1974, 26, 331-356.

(93) Born, G. V. R.; Bergquist, D.; Arfors, K.-E. Evidence for inhibition of platelet activation in blood by a drug effect on erythrocytes. Nature 1976, 259, 233-235.

(94) Sannanaik Vishwanath, B.; Manjunatha Kini, R.; Veerabasappa Gowda, T. Characterization of three edema-inducing phospholipase A2 enzymes from habu (Trimeresurus flavoviridis) venom and their interaction with the alkaloid aristolochic acid. Toxicon 1987, 25, 501515.

(95) Kondo, H.; Kondo, S.; Irezawa, H.; Murata, R.; Ohsaka, A. Studies on the Quantitative Method for Determination of Hemorrhagic Activity of Habu Snake Venom. Jpn. J. Med. Sci. Biol. 1960, 13, 43-51. 